\title{
Fixed-Time Disturbance Observer-Based Adaptive Finite-Time Guidance Law Design considering Impact Angle Constraint and Autopilot Dynamics
}

\author{
Yunjie Wu $\mathbb{D}^{1,2,3}$ Fei Ma $\mathbb{D}^{1,2,3}$ Xiaofei Yang $\mathbb{D}^{1,2,3}$ Siqi Wang $\mathbb{D}^{1,2,3}$ and Xiaodong Liu ${ }^{4}$ \\ ${ }^{1}$ State Key Laboratory of Virtual Reality Technology and Systems, Beihang University, Beijing 100191, China \\ ${ }^{2}$ School of Automation Science and Electrical Engineering, Beihang University, Beijing 100191, China \\ ${ }^{3}$ Science and Technology on Aircraft Control Laboratory, Beijing 100191, China \\ ${ }^{4}$ Beijing Aerospace Automatic Control Institute, Beijing 100854, China \\ Correspondence should be addressed to Fei Ma; mylovelylover26@163.com
}

Received 23 June 2021; Revised 21 July 2021; Accepted 1 August 2021; Published 14 August 2021

Academic Editor: Hamid Reza Karimi

Copyright (C) 2021 Yunjie Wu et al. This is an open access article distributed under the Creative Commons Attribution License, which permits unrestricted use, distribution, and reproduction in any medium, provided the original work is properly cited.

\begin{abstract}
This paper aims to establish an effective guidance law to accomplish the interception guidance mission for a missile intercepting a target with impact angle constraint and autopilot dynamics. To achieve this purpose, a fixed-time disturbance observer-based adaptive finite-time guidance law is presented. First, a fixed-time disturbance observer (FTDO) is designed to guarantee the fast estimation of the lumped disturbance caused by the target maneuver. Then, the FTDO-based adaptive integral sliding mode backstepping (AISMB) guidance law is constructed for the interception guidance problem. Besides, several adaptive laws are established to estimate the derivative of virtual control inputs, making the "differential explosion problem" of conventional backstepping get avoided. The finite-time convergence characteristic of the closed-loop system is analyzed by utilizing the Lyapunov stability theory. Finally, the simulation examples are conducted to demonstrate the effectiveness of the proposed composite guidance law.
\end{abstract}

\section{Introduction}

The high maneuverability of the targets can bring some great difficulties in the interception and defense in modern war [1]. Therefore, it is necessary to develop some innovative guidance methods [2] for the missiles to improve the interception efficiency against such targets. To achieve this goal, the missiles are usually required to complete the target interception at an expected impact angle while ensuring the small miss distance. By applying this guidance strategy, the efficiency of warheads and striking efficiency can be improved [3].

For a long time, the proportional navigation (PN) guidance law is one of the widely attractive methods which possesses simple calculation and easy implementation for the practical engineering [4]. On this basis, scholars have designed varieties of modified PN-based guidance laws, such as pure proportional navigation (PPN) guidance law [5], biased proportional navigation (BPN) guidance law [6], and generalized proportional navigation (GPN) guidance law [7]. By introducing an argument term to PN, Kim et al. [6] can intercept the target at the desired angle. In [7], the GPN is designed to achieve the guidance mission in the presence of small maneuvering and nonmaneuvering target. However, when facing a highly maneuvering target, few of these mentioned methods can simultaneously guarantee the small miss distance and desired impact angle. This disadvantage encourages the researchers to further develop some advanced methods for the guidance law, i.e., optimal control [8], sliding mode control [9], and disturbance observerbased control $[10,11]$.

The optimal control-based guidance law can achieve accurate attack at an expected impact angle under the premise of minor control energy. However, the design 
process of this guidance law depends on the estimation of time-to-go [12]. Though some scholars have proposed an excellent estimation of time-to-go and applied it to achieve the guidance mission with specified attack time [13], this guidance scheme cannot be suitable for other nonlinear schemes. As one of the most promising methods, the sliding mode control- (SMC-) based guidance law can provide the demanding of miss distance and terminal impact angle constraint without the prior knowledge of time-to-go. In addition, this method has great robustness to external disturbances and parameter uncertainties [14]. In [15], a guidance law for intercepting the target with evasive maneuver is proposed by applying the second-order sliding mode control method. Moreover, Ebrahimi et al. [16] combined optimal control and sliding mode control to construct a composite guidance law. Although these traditional SMC guidance laws can achieve robust guidance with simple forms, the state of the closed-loop system can only satisfy asymptotical stability. However, in some practical scenarios, the interception only lasts for several seconds. In this situation, the guidance law should have faster convergence speed, while the previous asymptotical stability cannot guarantee this property.

Subsequently, the finite-time stability theory [17] has been extended to the guidance and control of the spacecrafts for the purpose of addressing the aforesaid control issue. As known, the finite-time convergence theory-based controller can acquire the finite-time convergence property so as to drive the system state into the origin within a predefined convergence time [18]. Notably, the terminal sliding mode control is an excellent method to ensure this finite-time property. In [19], a finite-time convergent guidance law is presented to ensure that the line-of-sight (LOS) angular rate and LOS error converge to zero in finite time. Thus, the accurate interception is achieved, and the expected impact angle constraint is also satisfied. Based on the terminal sliding mode control theory, a guidance law [20] satisfying different initial conditions is designed to intercept the constant speed targets. Although abovementioned papers can solve the interception guidance problem with impact angle constraints, the target maneuvers are only considered as nonmoving or constant speed moving, which exhibits certain limitations in the practical engagements. Besides, traditional terminal sliding mode control may show the singularity when the sliding modes converge to zero [21]. Therefore, an adaptive nonsingular fast terminal sliding mode guidance law is developed by [22] to intercept the maneuvering targets with impact angle constraints and improve the singular problem of conventional terminal sliding mode control. Furthermore, one novel nonsingular terminal sliding mode control is designed to ensure that the missile strikes the target with the terminal impact angle constraint [23].

Strictly speaking, the autopilot dynamics of interceptor missiles can usually bring substantial influences to the actual guidance system [24], while most of the aforementioned studies do not focus on this issue. Thus, a finite-time guidance law considering the dynamics of the missile's autopilot as a first-order lag is designed in [25]. However, the autopilot lag would be more complicated in the actual situation and can usually be modeled as a second-order dynamics [26]. In [27], a robust continuous guidance law with the terminal angle constraint is proposed in the presence of second-order autopilot dynamics. In addition, Zhao et al. [28] presented one output feedback sliding mode guidance law by applying the sliding mode theory and dynamic surface control, in which the adaptive technique is utilized to acquire the estimation of target's maneuver.

To further improve the disturbance rejection capability, guidance laws based on the disturbance observer, i.e., nonlinear disturbance observer (NDO) [29], extended disturbance observer (ESO) [30], and sliding mode disturbance observer (SMO) [31], have been broadly applied to handle this issue. However, the convergence speed of the disturbance observers in aforesaid research studies is mainly depended on the observers' gains, and the transient process will be significantly deteriorated [32].

In recent years, fixed-time stability gets attractive and provides an effective strategy to construct the disturbance observer [33]. Theoretically speaking, the disturbance observers with fixed-time stability can guarantee that the estimation errors converge to zero in a prescribed fixed-time regardless of the initial conditions, indicating that these observers would obtain faster estimation [34]. In [35], a fixed-time differentiator-based disturbance observer is designed, which can ensure the exact estimation for the missile's control system. Besides, Zuo [36] extended the fixed-time stability technique to high-order systems and presented the general formulations of the fixed-time disturbance observer. Furthermore, the fixed-time disturbance observer (FTDO) is also developed in [37] so as to acquire the excellent observation of lumped disturbances. It is obvious that the fixed-time disturbance observer has shown quite impressive estimation performance [38].

Inspired by above discussions, this study attempts to employ the fixed-time disturbance observer to construct the composite adaptive finite time guidance law for intercepting highly maneuvering targets considering impact angle constraint and autopilot dynamics. Main contributions of this paper are summarized as follows:

(1) The fixed-time disturbance observer (FTDO) is utilized to estimate the lumped disturbance caused by target maneuver. Different from the most existing ESO [10] and SMO [31], the proposed FTDO can achieve fixed-time stability, and the convergence time is independent of the initial conditions.

(2) Based on the FTDO, an adaptive integral sliding mode backstepping (AISMB) guidance law is proposed to accomplish the interception mission, and the closed-loop guidance system can converge a small neighborhood around the origin in a finite time. The switching functions are introduced into the sliding mode reaching laws, which can guarantee the reaching property, meanwhile avoid the possible singularity problem. Moreover, the adopted adaptive laws can modify the control gains so as to reduce the control energy. 
(3) In contrast to the conventional backstepping methods [28], the proposed adaptive laws in AISMB are employed to estimate the derivative of virtual control inputs in the recursive design process, and the "explosion of terms" can be conquered accordingly.

The remaining part of this paper is organized as follows. The missile-target engagement motion equations, autopilot dynamics, and control-oriented model are described in Section 2. Section 3 presents the composite guidance scheme and the corresponding stability analysis of the closed-loop system. Numerical simulations are implemented in Section 4 so as to demonstrate the effectiveness of the proposed guidance law. Finally, some conclusion remarks are presented in Section 5.

\section{Problem Formulation}

2.1. Motion Kinematics and Relative Motion Dynamics. In this study, we consider the planar interception guidance problem with impact angle constraint and autopilot dynamics. The 2-dimensional (2D) homing engagement geometry for a missile intercepts a target, as depicted in Figure 1.

The velocity of the missile and target are represented by $V_{m}$ and $V_{t}$, respectively. $a_{m}$ and $a_{t}$ are used to represent the corresponding normal acceleration of the missile and target, respectively. $\gamma_{m}$ and $\gamma_{t}$ denote the flight path angle (FPA) of the missile and target, respectively. Besides, the LOS angle and relative disturbance between the missile and target are denoted by $\theta$ and $r$, respectively. Then, the kinematic engagement equations can be described by Kumar et al. [39]:

$$
\begin{aligned}
\dot{r} & =V_{t} \cos \theta_{t}-V_{m} \cos \theta_{m}, \\
r \dot{\theta} & =V_{t} \sin \theta_{t}-V_{m} \sin \theta_{m}, \\
\dot{\gamma}_{m} & =\frac{a_{m}}{V_{m}}, \\
\dot{\gamma}_{t} & =\frac{a_{t}}{V_{t}}
\end{aligned}
$$

where $\theta_{m}=\gamma_{m}-\theta, \theta_{t}=\gamma_{t}-\theta$.

Calculating the time derivatives of (1) and (2) yields

$$
\begin{aligned}
& \ddot{r}=r \dot{\theta}^{2}+a_{t} \sin \theta_{t}-a_{m} \sin \theta_{m}, \\
& \ddot{\theta}=-2 \frac{\dot{r}}{r} \dot{\theta}+\frac{\cos \theta_{t}}{r} a_{t}-\frac{\cos \theta_{m}}{r} a_{m} .
\end{aligned}
$$

Remark 1. Generally, the acceleration along the missile's velocity cannot be controlled in the terminal guidance phase, and only equation (6) is considered to design the guidance law. Besides, from (6), $\cos \theta_{m}=0$ will be satisfied when $\theta_{m}= \pm \pi / 2$, which can bring about control singularity and cause the failure of missile interception finally. However, if $\theta_{m}= \pm \pi / 2, \dot{\gamma}_{m}-\dot{\theta} \neq 0$ will hold. Thus, $\theta_{m}= \pm \pi / 2$ is not the

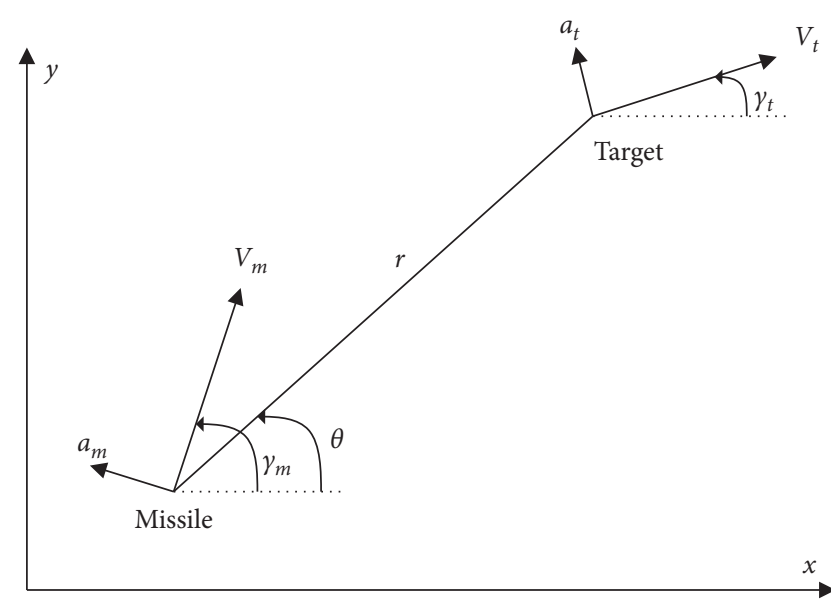

Figure 1: Two-dimensional engagement geometry.

stable equilibrium point, and the system trajectories will just cross this unstable point [20]. On this basis, $a_{m}$ can be applied to control the LOS angle $\theta$.

During the design process of the guidance law, we assume that the missile and the target are point masses moving in constant velocities $V_{m}$ and $V_{t}$, and the speed ratio of target-to-missile satisfies $v=V_{t} / V_{m}<1$. Besides, to avoid the possible singularity analyzed in Remark 1, equation (6) is rewritten as

$$
\ddot{\theta}=-2 \frac{\dot{r}}{r} \dot{\theta}-\frac{1}{r} a_{m}+\varphi,
$$

where $\varphi=\left(\cos \theta_{t} / r\right) a_{t}-\left(\cos \theta_{m} / r\right) a_{m}+(1 / r) a_{m}$.

When the missile hit the target, following relation exists [39]:

$$
V_{m} \sin \left(\gamma_{m f}-\theta_{f}\right)=V_{t} \sin \left(\gamma_{t f}-\theta_{f}\right),
$$

where $\gamma_{m f}, \gamma_{t f}$, and $\theta_{f}$ represent the final FPA of the missile, final FPA of the target, and final expected LOS angle, respectively.

Furthermore, with equation (2), $r \dot{\theta}=0$ will hold. Thus, the relation between the final LOS angle $\theta_{f}$ and designed impact angle $\theta_{\text {imp }}$ can be regarded as a one-to-one correspondence formulated by

$$
\theta_{f}=\gamma_{t f}-\arctan \left(\frac{\sin \theta_{\text {imp }}}{\cos \theta_{\text {imp }}-v}\right),
$$

where $\theta_{\text {imp }}=\gamma_{t f}-\gamma_{m f}$.

More specifically, when $\theta_{\text {imp }}=n \pi, n= \pm 1, \pm 2, \ldots$, the head-on and tail-chase intercept scenarios can be considered.

Remark 2. Basically, the missile can successfully achieve the interception by hit-to-kill impact, and the relative distance at the impact time is not equal to zero. Therefore, when $r \leq r_{0} \in\left[r_{\min }, r_{\max }\right]$ is satisfied, it can be considered that the missile has completed the interception mission.

Typically, the dynamics of autopilot can be modeled as the following second-order system [24]: 


$$
\ddot{a}_{m}=-2 \xi \omega_{n}-\omega_{n}^{2} \dot{a}_{m}+\omega_{n}^{2} a_{m c}
$$

where $\xi$ denotes the damping ratio, $\omega_{n}$ denotes the natural frequency, and $a_{m c}$ denotes the actual guidance command.

2.2. Control-Oriented Model. Considering autopilot dynamics (10), the acceleration command $a_{m c}$ can be designed to achieve the target interception at the expected LOS angle $\theta_{f}$. Therefore, let $x_{1}=\theta-\theta_{f}, \quad x_{2}=\dot{\theta}$, $x_{3}=a_{m}, x_{4}=\dot{a}_{m}$, and $u=a_{m c}$, and the control-oriented model can be described by

$$
\left\{\begin{array}{l}
\dot{x}_{1}=x_{2}, \\
\dot{x}_{2}=f_{2}+g_{2} x_{3}+d_{2}, \\
\dot{x}_{3}=x_{4}, \\
\dot{x}_{4}=f_{4}+g_{4} u,
\end{array}\right.
$$

where $f_{2}=-2(\dot{r} / r) x_{2}, g_{2}=-(1 / r), d_{2}=\varphi, f_{4}=-2 \xi \omega_{n} x_{3}-$ $\omega_{n}^{2} x_{3}^{2}$, and $g_{4}=\omega_{n}^{2}$.

Assumption 1. The target acceleration and its derivative are bounded, that is, $\left|a_{t}\right| \leq a_{t}^{\max }$ and $\left|\dot{a}_{t}\right| \leq \dot{a}_{t}^{\max }$.

Assumption 2. The derivative of the disturbance $\dot{d}_{2}$ is bounded and satisfies $\left|\dot{d}_{2}\right| \leq d_{2 \max }$.

Finally, the purpose of this guidance law design problem is to ensure the small miss distance and small LOS error by driving the state $x_{1}$ and $x_{2}$ to the small neighborhood of zero in finite time.

\section{Guidance Law Design and Analysis}

In this section, the design process of the guidance scheme is described in detail. Specifically, the fixed-time disturbance observer is employed to estimate the lumped disturbance. Then, the FTDO-based guidance law is developed by combing the adaptive theory, integral sliding mode control, and backstepping control. The control structure of the guidance scheme is shown in Figure 2.

Notation 1. In this paper, $\operatorname{sig}(x)^{p}=\operatorname{sign}(x)|x|^{p}$, where $\operatorname{sign}(x)$ is the sign function.

Before the conduction of the guidance law, some necessary definitions and lemmas are introduced as follows.

Definition 1 (see [40]). Consider the system

$$
\begin{aligned}
\dot{\mathbf{x}} & =\mathbf{f}(\mathbf{x}, t), \\
\mathbf{f}(0, t) & =0, \quad \mathbf{x} \in \mathbb{R}^{n},
\end{aligned}
$$

where $\mathbf{f}: \mathbf{N}_{0} \longrightarrow \mathbb{R}^{n}$ is continuous on the open neighborhood $\mathbf{N}_{0}$ of the origin $\mathbf{x}=0$. For any $t_{0}$, given the initial state $\mathbf{x}_{0}$, which relies on the settling time $T\left(\mathbf{x}_{0}\right)$, then system (12) exists in a solution $\mathbf{x}=\varphi\left(t, t_{0}, \mathbf{x}_{0}\right)$. If $t \in\left[t_{0}, T\left(\mathbf{x}_{0}\right)\right)$, $\varphi\left(t, t_{0}, \mathbf{x}_{0}\right) \in \mathbf{N} /\{0\}$ with $\lim _{t \longrightarrow T\left(\mathbf{x}_{0}\right)} \varphi\left(t, t_{0}, \mathbf{x}_{0}\right)=0$ holds, and if $t>T\left(\mathbf{x}_{0}\right), \varphi\left(t, t_{0}, \mathbf{x}_{0}\right)=0$ will hold. The origin of (12) possesses locally finite-time stability in the neighborhood
$\mathbf{N} \subseteq \mathbf{N}_{0}$ if it is also Lyapunov stable. This solution is said to be globally finite-time stable if $\mathbf{N}=\mathbf{N}_{0}=\mathbb{R}^{n}$.

Remark 3. From above analysis, the finite-time stability will imply the asymptotic stability of the origin, and the convergence time can be guaranteed by a prescribed time $T=T\left(\mathbf{x}_{0}\right)$. If the system initial state $\mathbf{x}_{0}$ can be obtained, the settling time can be evaluated by $T\left(\mathbf{x}_{0}\right)$, and the system can achieve finite-time stability. However, if the system state $\mathbf{x}_{0}$ is not available, the settling time cannot be estimated by $T\left(\mathbf{x}_{0}\right)$ in advance. In this situation, it is possible that $T\left(\mathbf{x}_{0}\right)$ will become large if the initial state is very large. Thus, to remedy this shortcoming, Polyakov [41] proposes the fixed-time stability which can guarantee that the settling time $T=T\left(\mathbf{x}_{0}\right)$ will be independent of the initial condition $\mathbf{x}_{0}$.

Definition 2 (see [41]). Based on Definition 1, the origin of (12) is said to be fixed-time stable if it satisfies globally finitetime stable and the settling time $T\left(\mathbf{x}_{0}\right)$ is bounded, that is, for any initial state $\mathbf{x}_{0}$, there exists $T_{\max }>0$ such that $T\left(\mathbf{x}_{0}\right)<T_{\max }$.

Remark 4. The settling time of fixed-time stability is regardless of the initial state, while finite time is the opposite. This property implies that the convergence time can be determined by a prescribed manner.

Lemma 1 (see [21]). For system (12) with continuous positive definite function $V(\mathbf{x})$, if the following equation

$$
\dot{V}(\mathbf{x}) \leq-\tau V(\mathbf{x})^{\eta}
$$

where $\tau>0$ and $0<\eta<1$, holds, then the system will converge to the origin in finite time bounded by

$$
t \leq \frac{V^{1-\eta}\left(\mathbf{x}_{0}\right)}{\tau(1-\eta)}
$$

Lemma 2 (see [1]). Similarly, if $V(\mathbf{x})$ defined in Lemma 1 satisfies

$$
\dot{V}(\mathbf{x}) \leq-a V(\mathbf{x})-b V(\mathbf{x})^{\gamma},
$$

where $a>0, b>0$, and $0<\gamma<1$, the system converges to the origin in finite time bounded by

$$
t \leq \frac{1}{a(1-\gamma)} \ln \frac{a V\left(\mathbf{x}_{0}\right)^{1-\gamma}+b}{b} .
$$

Lemma 3 (see [28]). For positive real numbers $y_{1}, y_{2}, \ldots, y_{n}$ and $0<p<1$, the following inequality is satisfied:

$$
\left(y_{1}^{2}+y_{2}^{2}+\cdots+y_{n}^{2}\right)^{p} \leq\left(y_{1}^{p}+y_{2}^{p}+\cdots+y_{n}^{p}\right)^{2} .
$$

Lemma 4 (see $[42,43]$ ). For the following system,

$$
s=\dot{e}_{n}+k_{n} \operatorname{sig}\left(e_{n-1}\right)^{\alpha_{n}}+\cdots+k_{1} \operatorname{sig}\left(e_{1}\right)^{\alpha_{1}},
$$




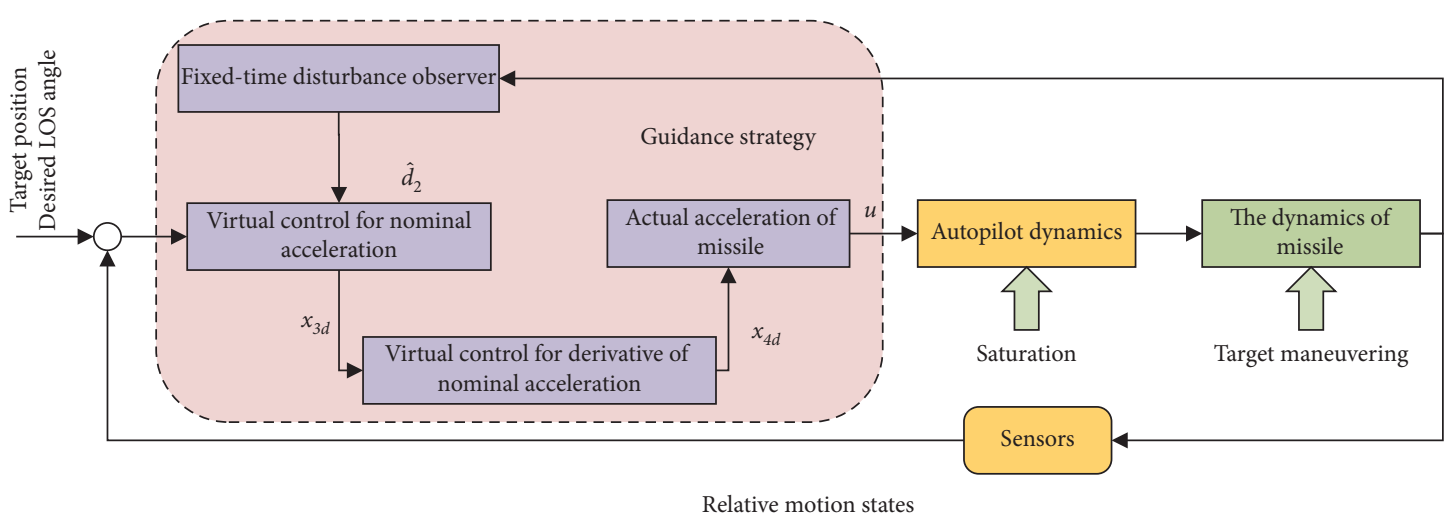

FIGURE 2: Control diagram of the composite guidance strategy

where $k_{i}$ and $\alpha_{i} \quad(i=1,2, \ldots, n)$ are constants, $\dot{e}_{i}=e_{i+1},(i=1,2, \ldots, n-1)$. If $k_{i}$ is selected such that the polynomial $p^{n}+k_{n} p^{n-1}+\cdots+k_{2} p+k_{1}$ is Hurwitz and $\alpha_{i}$ are positive constants satisfying

$$
\left\{\begin{array}{l}
\alpha_{1}=\alpha \\
n=1 \\
\alpha_{i-1}=\frac{\alpha_{i} \alpha_{i+1}}{2 \alpha_{i+1}-\alpha_{i}}, \quad i=2, \ldots, n,
\end{array}\right.
$$

where $\alpha_{n+1}=1$ and $\alpha_{n}=\alpha \in(0,1)$, then the system will converge to its equilibrium point in finite time. When $n=2$, the settling time can be estimated by

$$
t \leq \frac{\left(3+\alpha_{1}\right) V_{0}^{\left(1-\alpha_{1}\right) /\left(3+\alpha_{1}\right)}}{K\left(1-\alpha_{1}\right)}
$$

where $V_{0}$ denotes the initial value of the selected Lyapunov function $V$ and $K$ is a positive constant.

3.1. The Design of Fixed-Time Disturbance Observer. As is known to all, the lumped disturbance $d_{2}$ will degrade the performance of the missile guidance and control system. Inspired by [44], a disturbance observer with fixed-time convergence characteristics is proposed to estimate the disturbances. This fixed-time disturbance observer can drive the estimation error to a small neighborhood of the origin within a bounded time regardless of the initial state. Therefore, we can obtain following theorem.

Theorem 1. For guidance system (11), the unknown term $d_{2}$ satisfies $\left|\dot{d}_{2}\right|<L_{2}$, where $L_{2}$ is a positive constant. The fixedtime disturbance observer can be described as

$$
\left\{\begin{array}{l}
\dot{\hat{x}}_{2}=f_{2}+g_{2} x_{3}+\hat{d}_{2}, \\
\widehat{d}_{2}=k_{21} \operatorname{sig}\left(e_{21}\right)^{p}+k_{22} \operatorname{sig}\left(e_{21}\right)^{q}+\int_{0}^{t} k_{23} \operatorname{sign}\left(e_{21}(\tau)\right) \mathrm{d} \tau,
\end{array}\right.
$$

where $0<p<1, q>1, \hat{x}_{2}$ is the estimation of $x_{2}, e_{21}=x_{2}-\hat{x}_{2}$ represents the estimate error, and $k_{2 j}(j=1,2$, and 3$)$ are all positive designed parameters that satisfy the follow conditions:

$$
\left\{\begin{array}{l}
M_{2}=k_{23}+L_{2}, \\
m_{2}=k_{i 3}-L_{2}, \\
h_{2}=\frac{1}{k_{21}}+\left(\frac{2 e}{m_{2} k_{21}}\right)^{1 / 3}, \\
k_{23}>L_{2}, \\
k_{21} h>M_{2},
\end{array}\right.
$$

with e representing the nature constant.

Proof. Taking the derivative of $e_{21}$, one can obtain

$$
\begin{aligned}
\dot{e}_{21} & =\dot{x}_{2}-\dot{\hat{x}}_{2}=d_{2}-\hat{d}_{2} \\
& =d_{2}-k_{21} \operatorname{sig}\left(e_{21}\right)^{p}-k_{22} \operatorname{sig}\left(e_{21}\right)^{q}-\int_{0}^{t} k_{23} \operatorname{sign}\left(e_{21}(\tau)\right) \mathrm{d} \tau .
\end{aligned}
$$

Define $e_{22}=d_{2}-\int_{0}^{t} k_{23} \operatorname{sign}\left(e_{21}(\tau)\right) \mathrm{d} \tau$, and equation (23) can be rewritten as

$$
\dot{e}_{21}=-k_{21} \operatorname{sig}\left(e_{21}\right)^{p}-k_{22} \operatorname{sig}\left(e_{21}\right)^{q}+e_{22} .
$$

Then, calculating the derivative of $e_{22}$, one has

$$
\dot{e}_{22}=-k_{33} \operatorname{sign}\left(e_{21}\right)+\dot{d}_{2} \text {. }
$$

According to equations (24) and (25), the following error dynamics can be obtained:

$$
\left\{\begin{array}{l}
\dot{e}_{21}=-k_{21} \operatorname{sig}\left(e_{21}\right)^{p}-k_{22} \operatorname{sig}\left(e_{21}\right)^{q}+e_{22}, \\
\dot{e}_{22}=-k_{33} \operatorname{sign}\left(e_{21}\right)+\dot{d}_{2} .
\end{array}\right.
$$

On the basis of the result in [44], if the observer gains satisfy condition (22), $e_{21}$ and $e_{22}$ can uniformly converge to the origin within a fixed-time bounded by 


$$
t_{o} \leq\left(\frac{1}{k_{22}} \frac{1}{q-1}-\frac{1}{k_{21}} \frac{1}{1-p}\right)\left(1+\frac{M_{2}}{\left(1-M_{2} h_{2} / k_{21}\right) m_{2}}\right) .
$$

This result means that $e_{21}=0$ and $e_{22}=0$ for $t>t_{o}$. Recalling (23), it can be obtained that $\dot{e}_{21}=d_{2}-\widehat{d}_{2}=0$. Therefore, the equivalent disturbance can be exactly estimated after $t>t_{o}$. This completes the proof of Theorem 1 .

Remark 5. Compared with SMO and ESO, the applied FTDO can achieve the fixed-time stability, and the settling time is uniformly bounded and independent of the initial state. Besides, as shown in equation (21), the sign function is integrated in the integral term, which can guarantee the continuity of the estimation value and eliminate the chattering problem. This characteristic will be confirmed by the subsequent simulation results.

3.2. FTDO-Based Adaptive Integral Sliding Mode Backstepping Guidance Law Design. As shown in the previous section, the estimation of unknown disturbance can be obtained by the proposed FTDO in a short time regardless of the initial conditions. Based on this merit, the following composite guidance law is developed in detail.

3.2.1. Finite Time Integral Siding Mode Control. Generally speaking, the small miss distance and the desired LOS angle can be satisfied by driving $x_{1}$ and $x_{2}$ to zero [17]. Therefore, the sliding surface should contain $x_{1}$ and $x_{2}$. In this situation, we introduce a novel integral sliding surface $s_{2}$ defined as

$$
s_{2}=x_{2}-x_{2}(0)+\int \lambda_{11} \operatorname{sig}\left(x_{1}\right)^{\alpha_{1}}+\lambda_{12} \operatorname{sig}\left(x_{2}\right)^{\alpha_{2}} \mathrm{~d} \tau,
$$

where $\lambda_{11}>0, \lambda_{12}>0,0<\alpha_{1}=\alpha<1$, and $\alpha_{2}=\left(2 \alpha_{1} / \alpha_{1}+1\right)$.

Combing the second equation of (11) and (28), it is clear that

$$
\begin{aligned}
\dot{s}_{2} & =\dot{x}_{2}+\lambda_{11} \operatorname{sig}\left(x_{1}\right)^{\alpha_{1}}+\lambda_{12} \operatorname{sig}\left(x_{2}\right)^{\alpha_{2}} \\
& =f_{2}+g_{2} x_{3}+d_{2}+\lambda_{11} \operatorname{sig}\left(x_{1}\right)^{\alpha_{1}}+\lambda_{12} \operatorname{sig}\left(x_{2}\right)^{\alpha_{2}} .
\end{aligned}
$$

To stabilize the subsystem of $x_{2}$, the virtual control law can be designed as

$$
x_{3 d}=g_{2}^{-1}\left(-f_{2}-\lambda_{11} \operatorname{sig}\left(x_{1}\right)^{\alpha_{1}}-\lambda_{12} \operatorname{sig}\left(x_{2}\right)^{\alpha_{2}}-k_{2} F_{2}\left(s_{2}\right)-\hat{\lambda}_{2} s_{2}-\hat{d}_{2}\right),
$$

where $k_{2}>0$ is the designed parameter, $\widehat{d}_{2}$ is estimated by the designed FTDO, and $F_{2}\left(s_{2}\right)$ is constructed as

$$
F_{2}\left(s_{2}\right)=\left\{\begin{array}{l}
\operatorname{sig}\left(s_{2}\right)^{\beta}, \quad\left|s_{2}\right| \geq \omega_{2}, \\
\tau_{21} s_{2}+\tau_{22} s_{2}^{2} \operatorname{sign}\left(s_{2}\right), \quad\left|s_{2}\right|<\varpi_{2},
\end{array}\right.
$$

with $0<\beta<1$, where $\Phi_{2}$ is a small enough positive constant, $\tau_{21}=(2-\beta) \omega_{2}^{\beta-1}, \tau_{22}=(1-\beta) \omega_{2}^{\beta-2}$, and $\widehat{\lambda}_{2}$ is an adaptive gain designed as

$$
\dot{\hat{\lambda}}_{2}= \begin{cases}\kappa_{2} s_{2}^{2}-\sigma_{2} \hat{\lambda}_{2}, & \left|s_{2}\right| \geq \delta_{2} \\ 0, & \text { else, }\end{cases}
$$

with $\kappa_{2}>0, \sigma_{2}>0$, and $\delta_{2}>0$.

Remark 6. By introducing switching function (31) [45], the potential singularity that appeared in the derivative of term $\operatorname{sig}\left(s_{2}\right)^{\beta}$ will get avoided. For the case $\left|s_{2}\right| \geq \omega_{2}, \dot{F}_{2}\left(s_{2}\right)=$ $\beta\left|s_{2}\right|^{\beta-1} \dot{s}_{2}$ is nonsingular, while for the case $\left|s_{2}\right|<\Phi_{2}$, $\dot{F}_{2}\left(s_{2}\right)=\tau_{21} \dot{s}_{2}+2 \tau_{22}\left|s_{2}\right| \dot{s}_{2}$ will also not to be singular when $s_{2}=0$ and $\dot{s}_{2} \neq 0$. However, the subsystem will approach zero with worse convergence property when $\left|s_{2}\right|<\omega_{2}$. Thus, we should select small enough to guarantee the power rate reaching law property, meanwhile avoid the singularity problem.

Define the state error of $x_{3}$ as

$$
s_{3}=x_{3}-x_{3 d} \text {. }
$$

Then, substituting (33) and (30) into (29), one can obtain that

$$
\begin{aligned}
\dot{s}_{2} & =f_{2}+g_{2}\left(x_{3 d}+s_{3}\right)+d_{2}+\lambda_{11} \operatorname{sig}\left(x_{1}\right)^{\alpha_{1}}+\lambda_{12} \operatorname{sig}\left(x_{2}\right)^{\alpha_{2}} \\
& =f_{2}+g_{2} s_{3}+g_{2} x_{3 d}+d_{2}+\lambda_{11} \operatorname{sig}\left(x_{1}\right)^{\alpha_{1}}+\lambda_{12} \operatorname{sig}\left(x_{2}\right)^{\alpha_{2}} \\
& =-k_{2} F_{2}\left(s_{2}\right)-\hat{\lambda}_{2} s_{2}+d_{2}-\widehat{d}_{2}+g_{2} s_{3} .
\end{aligned}
$$

3.2.2. Backstepping Strategy. The backstepping scheme contains a step-by-step construction of the new subsystem by the state error $s_{i}=x_{i}-x_{i d}(i=3,4)$, where $x_{i d}$ is the desired control command for state $x_{i}$ [29]. Based on this strategy, calculating the derivative of $s_{3}$ yields

$$
\dot{s}_{3}=\dot{x}_{3}-\dot{x}_{3 d}=x_{4}-\dot{x}_{3 d}
$$

It is obvious that the derivative of $x_{3 d}$ should be compensated in the subsequent design step. However, it is difficult to calculate the exact value of $\dot{x}_{3 d}$, and the complexity will increase as the order of the system increases. In order to solve this "differential explosion" problem, $\dot{x}_{i d}$ will be considered as an uncertain term with the following reasonable assumption and finally resolved by the predesigned adaptive laws.

Assumption 3 (see [29]). $x_{3 d}$ and $x_{4 d}$ are bounded, satisfying $\left|x_{i d}\right| \leq \chi_{i}(i=3$ and 4$)$ where $\chi_{3}$ and $\chi_{4}$ are all positive constants.

Thus, the virtual control $x_{4 d}$ can be designed as

$$
x_{4 d}=-\frac{\widehat{\rho}_{3} s_{3}}{2 \varepsilon_{3}^{2}}-k_{3} F_{3}\left(s_{3}\right)-\widehat{\lambda}_{3} s_{3}-g_{2} s_{2}
$$

where $k_{3}>0$ is the designed parameter and $F_{3}\left(s_{3}\right)$ is constructed as

$$
F_{3}\left(s_{3}\right)=\left\{\begin{array}{l}
\operatorname{sig}\left(s_{3}\right)^{\beta}, \quad\left|s_{3}\right| \geq \varpi_{3}, \\
\tau_{31} s_{3}+\tau_{32} s_{3}^{2} \operatorname{sign}\left(s_{3}\right), \quad\left|s_{3}\right|<\Phi_{3},
\end{array}\right.
$$


with $\Phi_{3}$ is a small enough positive constant, where $\tau_{31}=(2-\beta) \oplus_{3}^{\beta-1}, \tau_{32}=(1-\beta) \omega_{3}^{\beta-2}$, and $\hat{\lambda}_{3}$ is an adaptive gain designed as

$$
\dot{\hat{\lambda}}_{3}= \begin{cases}\kappa_{3} s_{3}^{2}-\sigma_{3} \hat{\lambda}_{3}, & \left|s_{3}\right| \geq \delta_{3}, \\ 0, & \text { else, }\end{cases}
$$

with $\kappa_{2}>0, \sigma_{2}>0$, and $\delta_{2}>0 . \hat{\rho}_{3}$ is an adaptive parameter designed as

$$
\dot{\hat{\rho}}_{3}=\frac{\gamma_{3} s_{3}^{2}}{2 \varepsilon_{3}^{2}}-\xi_{3} \hat{\rho}_{3},
$$

with $\varepsilon_{3}>0, \gamma_{3}>0$, and $\xi_{3}>0$.

Substituting (36) into (35), one obtains

$$
\begin{aligned}
\dot{s}_{3} & =x_{4}-\dot{x}_{3 d}=x_{4 d}+s_{4}-\dot{x}_{3 d} \\
& =-\frac{\hat{\rho}_{3} s_{3}}{2 \varepsilon_{3}^{2}}-k_{3} F_{3}\left(s_{3}\right)-\hat{\lambda}_{3} s_{3}-g_{2} s_{2}-\dot{x}_{3 d}+s_{4} .
\end{aligned}
$$

Similarly, the state error for $x_{4}$ is defined as

$$
s_{4}=x_{4}-x_{4 d} \text {. }
$$

Calculating the time derivative of $s_{4}$ yields

$$
\dot{s}_{4}=\dot{x}_{4}-\dot{x}_{4 d}=f_{4}+g_{4} u+d_{4}-\dot{x}_{4 d} .
$$

Then, the actual control law is designed as

$$
u=g_{4}^{-1}\left(-f_{4}-s_{3}-\frac{\hat{\rho}_{4} s_{4}}{2 \varepsilon_{4}^{2}}-k_{4} F_{4}\left(s_{4}\right)-\hat{\lambda}_{4} s_{4}\right),
$$

where $k_{4}>0$ is the designed parameter and $F_{4}\left(s_{4}\right)$ is constructed as

$$
F_{4}\left(s_{4}\right)=\left\{\begin{array}{l}
\operatorname{sig}\left(s_{4}\right)^{\beta}, \quad\left|s_{4}\right| \geq \varpi_{4}, \\
\tau_{41} s_{4}+\tau_{42} s_{4}^{2} \operatorname{sign}\left(s_{4}\right), \quad\left|s_{4}\right|<\varpi_{4},
\end{array}\right.
$$

with $\Phi_{4}$ is a small enough positive constant, where $\tau_{41}=$ $(2-\beta) \omega_{4}^{\beta-1}$ and $\tau_{42}=(1-\beta) \omega_{4}^{\beta-2} . \widehat{\lambda}_{4}$ is an adaptive gain designed as

$$
\dot{\hat{\lambda}}_{4}= \begin{cases}\kappa_{4} s_{4}^{2}-\sigma_{4} \hat{\lambda}_{4}, & \left|s_{4}\right| \geq \delta_{4}, \\ 0, & \text { else, }\end{cases}
$$

with $\kappa_{4}>0, \sigma_{4}>0$, and $\delta_{4}>0 . \hat{\rho}_{4}$ is an adaptive parameter designed as

$$
\dot{\hat{\rho}}_{4}=\frac{\gamma_{4} s_{4}^{2}}{2 \varepsilon_{4}^{2}}-\xi_{4} \hat{\rho}_{4},
$$

with $\varepsilon_{3}>0, \gamma_{3}>0$, and $\xi_{3}>0$.

Substituting (43) into (42), one has

$$
\begin{aligned}
\dot{s}_{4} & =f_{4}+g_{4} u+d_{4}-\dot{x}_{4 d} \\
& =-s_{3}-\frac{\hat{\rho}_{4} s_{4}}{2 \varepsilon_{4}^{2}}-k_{4} F_{4}\left(s_{4}\right)-\widehat{\lambda}_{4} s_{4}-\dot{x}_{4 d} .
\end{aligned}
$$

Based on the above design steps, the proposed FTDObased guidance law can be summarized as

$$
\left\{\begin{array}{l}
s_{2}=x_{2}-x_{2}(0)+\int \lambda_{11} \operatorname{sig}\left(x_{1}\right)^{\alpha_{1}}+\lambda_{12} \operatorname{sig}\left(x_{2}\right)^{\alpha_{2}} \mathrm{~d} \tau \\
x_{3 d}=g_{2}^{-1}\left(-f_{2}-\lambda_{11} \operatorname{sig}\left(x_{1}\right)^{\alpha_{1}}-\lambda_{12} \operatorname{sig}\left(x_{2}\right)^{\alpha_{2}}-k_{2} F_{2}\left(s_{2}\right)-\hat{\lambda}_{2} s_{2}-\widehat{d}_{2}\right) \\
s_{3}=x_{3}-x_{3 d}, \\
x_{4 d}=-\frac{\hat{\rho}_{3} s_{3}}{2 \varepsilon_{3}^{2}}-k_{3} F_{3}\left(s_{3}\right)-\hat{\lambda}_{3} s_{3}-g_{2} s_{2}, \\
s_{4}=x_{4}-x_{4 d}, \\
u=g_{4}^{-1}\left(-f_{4}-s_{3}-\frac{\hat{\rho}_{4} s_{4}}{2 \varepsilon_{4}^{2}}-k_{4} F_{4}\left(s_{4}\right)-\hat{\lambda}_{4} s_{4}\right)
\end{array}\right.
$$

with functions $F_{i}\left(s_{i}\right)(i=2,3$, and 4$)$ defined as

$$
F_{i}\left(s_{i}\right)=\left\{\begin{array}{l}
\operatorname{sig}\left(s_{i}\right)^{\beta}, \quad\left|s_{i}\right| \geq \varpi_{i}, \\
\tau_{i 1} s_{2}+\tau_{i 2} s_{i}^{2} \operatorname{sign}\left(s_{i}\right), \quad\left|s_{i}\right|<\Phi_{i},
\end{array}\right.
$$

and adaptive laws: $\dot{\hat{\lambda}}_{i}=\left\{\begin{array}{ll}\kappa_{i} s_{i}^{2}-\sigma_{i} \widehat{\lambda}_{i}, & \left|s_{i}\right| \geq \delta_{i}, \quad \\ 0, & \text { else, }\end{array} \quad i=2,3\right.$, and 4 
Theorem 2. For guidance system (11) with proposed AISMB guidance law (48), FTDO (21), as well as adaptive laws (50) and (51), the closed-loop system can achieve finite time stability and the sliding modes $s_{i}(i=2,3$, and 4$)$ and states $x_{1}$ and $x_{2}$ are guaranteed to converge to the small neighborhood around the origin in finite time.

Proof. Selecting the Lyapunov candidate as

$$
V_{1}=\frac{1}{2} \sum_{i=2}^{4} s_{i}^{2}+\frac{1}{2} \sum_{i=2}^{4} \frac{\tilde{\lambda}_{i}^{2}}{\kappa_{i}}+\frac{1}{2} \sum_{j=3,4} \frac{\tilde{\rho}_{j}^{2}}{\gamma_{j}}
$$

where $\tilde{\lambda}_{i}=\bar{\lambda}_{i}-\hat{\lambda}, \tilde{\rho}_{j}=\bar{\rho}_{j}-\widehat{\rho}_{j}, \bar{\lambda}_{i}>0$, and $\bar{\rho}_{i}>0$.

Its time derivative is

$$
\dot{V}_{1}=\sum_{i=2}^{4} s_{i} \dot{s}_{i}-\sum_{i=2}^{4} \frac{\tilde{\lambda}_{i} \dot{\hat{\lambda}}_{i}}{\kappa_{i}}-\sum_{j=3,4} \frac{\widetilde{\rho}_{j} \dot{\hat{\rho}}_{j}}{\gamma_{j}} .
$$

Substituting equations (34), (40), and (47) into (53), one obtains

$$
\begin{aligned}
\dot{V}_{1}= & s_{2}\left(-k_{2} F_{2}\left(s_{2}\right)-\hat{\lambda}_{2} s_{2}+d_{2}-\widehat{d}_{2}+g_{2} s_{3}\right) \\
& +s_{3}\left(-\frac{\hat{\rho}_{3} s_{3}}{2 \varepsilon_{3}^{2}}-k_{3} F_{3}\left(s_{3}\right)-\hat{\lambda}_{3} s_{3}-g_{2} s_{2}-\dot{x}_{3 d}+s_{4}\right) \\
& +s_{4}\left(-s_{3}-\frac{\hat{\rho}_{4} s_{4}}{2 \varepsilon_{4}^{2}}-k_{4} F_{4}\left(s_{4}\right)-\hat{\lambda}_{4} s_{4}-\dot{x}_{4 d}\right)-\sum_{i=2}^{4} \frac{\tilde{\lambda}_{i}}{\kappa_{i}}-\sum_{j=3,4} \frac{\tilde{\rho}_{j} \dot{\hat{\rho}}_{j}}{\gamma_{j}}
\end{aligned}
$$

where $e_{2}=d_{2}-\widehat{d}_{2}$.

Since $\omega_{i}$ are sufficient small positive constants, (54) can be rewritten as

$$
\begin{aligned}
\dot{V}_{1}= & -\sum_{i=2}^{4} k_{i}\left|s_{i}\right|^{\beta+1}-\sum_{i=2}^{4} \hat{\lambda}_{i} s_{i}^{2}+s_{2} e_{2}-\frac{\hat{\rho}_{3} s_{3}^{2}}{2 \varepsilon_{3}^{2}}-\dot{x}_{3 d} s_{3}-\frac{\hat{\rho}_{4} s_{4}^{2}}{2 \varepsilon_{4}^{2}}-s_{4} \dot{x}_{4 d} \\
& -\sum_{i=2}^{4} \frac{\tilde{\lambda}_{i} \dot{\hat{\lambda}}_{i}}{\kappa_{i}}-\sum_{j=3,4} \frac{\tilde{\rho}_{j} \dot{\hat{\rho}}_{j}}{\gamma_{j}} .
\end{aligned}
$$

With adaptive laws (50) and (51), (55) becomes

$$
\begin{aligned}
& \dot{V}_{1}=-\sum_{i=2}^{4} k_{i}\left|s_{i}\right|^{\beta+1}-\sum_{i=2}^{4} \hat{\lambda}_{i} s_{i}^{2}+s_{2} e_{2}-\frac{\hat{\rho}_{3} s_{3}^{2}}{2 \varepsilon_{3}^{2}}-\dot{x}_{3 d} s_{3}-\frac{\widehat{\rho}_{4} s_{4}^{2}}{2 \varepsilon_{4}^{2}}-s_{4} \dot{x}_{4 d} \\
& -\sum_{i=2}^{4} \frac{\tilde{\lambda}_{i}}{\kappa_{i}}\left(\kappa_{i} s_{i}^{2}-\sigma_{i} \hat{\lambda}_{i}\right)-\sum_{j=3,4} \frac{\tilde{\rho}_{j}}{\gamma_{j}}\left(\frac{\gamma_{j} s_{j}^{2}}{2 \varepsilon_{j}^{2}}-\xi_{j} \widehat{\rho}_{j}\right) \\
& =-\sum_{i=2}^{4} k_{i}\left|s_{i}\right|^{\beta+1}+\sum_{i=2}^{4}\left(-\bar{\lambda}_{i} s_{i}^{2}+\frac{\sigma_{i}}{\kappa_{i}} \widetilde{\lambda}_{i} \widehat{\lambda}_{i}\right)+s_{2} e_{2}+\sum_{j=3,4}\left(-x_{j d} s_{j}-\frac{\bar{\rho}_{j} s_{j}^{2}}{2 \varepsilon_{j}^{2}}+\frac{\xi_{j}}{\gamma_{j}} \widetilde{\rho}_{j} \widehat{\rho}_{j}\right) .
\end{aligned}
$$

According to Assumption 3, one has 


$$
\begin{aligned}
\dot{V}_{1} & \leq-\sum_{i=2}^{4} k_{i}\left|s_{i}\right|^{\beta+1}+\sum_{i=2}^{4}\left(-\bar{\lambda}_{i} s_{i}^{2}+\frac{\sigma_{i}}{\kappa_{i}} \widetilde{\lambda}_{i} \hat{\lambda}_{i}\right)+s_{2} e_{2}+\sum_{j=3,4}\left(\left|-x_{j d}\right|\left|s_{j}\right|-\frac{\bar{\rho}_{j} s_{j}^{2}}{2 \varepsilon_{j}^{2}}+\frac{\xi_{j}}{\gamma_{j}} \widetilde{\rho}_{j} \widehat{\rho}_{j}\right) \\
& \leq-\sum_{i=2}^{4} k_{i}\left|s_{i}\right|^{\beta+1}+\sum_{i=2}^{4}\left(-\bar{\lambda}_{i} s_{i}^{2}+\frac{\sigma_{i}}{\kappa_{i}} \widetilde{\lambda}_{i} \hat{\lambda}_{i}\right)+s_{2} e_{2}+\sum_{j=3,4}\left(\chi_{j}\left|s_{j}\right|-\frac{\bar{\rho}_{j} s_{j}^{2}}{2 \varepsilon_{j}^{2}}+\frac{\xi_{j}}{\gamma_{j}} \widetilde{\rho}_{j} \widehat{\rho}_{j}\right)
\end{aligned}
$$

Noting $\left|s_{j}\right| \chi_{j} \leq\left(\left|s_{j}\right|^{2} / 2 \varepsilon_{j}^{2}\right) \chi_{j}^{2}+\left(\varepsilon_{j}^{2} / 2\right)$ and $s_{2} e_{2} \leq$ $\left(1 / 2 v_{2}\right) s_{2}^{2}+\left(v_{2} / 2\right) e_{2}^{2}\left(v_{2}\right.$ is a positive constant which is only used for analysis), one can get

$$
\begin{aligned}
\dot{V}_{1} \leq & -\sum_{i=2}^{4} k_{i}\left|s_{i}\right|^{\beta+1}+\sum_{i=2}^{4}\left(-\bar{\lambda}_{i} s_{i}^{2}+\frac{\sigma_{i}}{\kappa_{i}} \widehat{\lambda}_{i} \widehat{\lambda}_{i}\right)+\frac{1}{2 \nu_{2}} s_{2}^{2}+\frac{\nu_{2}}{2} e_{2}^{2} \\
& +\sum_{j=3,4}\left(\frac{\left|s_{j}\right|^{2}}{2 \varepsilon_{j}^{2}} \chi_{j}^{2}+\frac{\varepsilon_{j}^{2}}{2}-\frac{\bar{\rho}_{j} s_{j}^{2}}{2 \varepsilon_{j}^{2}}+\frac{\xi_{j}}{\gamma_{j}} \widetilde{\rho}_{j} \widehat{\rho}_{j}\right) .
\end{aligned}
$$

By the definition of $\widetilde{\lambda}_{i}$ and $\widetilde{\rho}_{j}$, it can be obtained that

$$
\left\{\begin{array}{l}
\widetilde{\lambda}_{i} \widehat{\lambda}_{i} \leq-\frac{1}{2} \widetilde{\lambda}_{i}^{2}+\frac{1}{2} \bar{\lambda}_{i}^{2}, \\
\tilde{\rho}_{j} \widehat{\rho}_{j} \leq-\frac{1}{2} \widetilde{\rho}_{j}^{2}+\frac{1}{2} \bar{\rho}_{j}^{2} .
\end{array}\right.
$$

$$
\begin{aligned}
\dot{V}_{1} \leq & -\sum_{i=2}^{4} k_{i}\left|s_{i}\right|^{\beta+1}-\bar{\lambda}_{2} s_{2}^{2}-\left(\bar{\lambda}_{3}+\frac{\bar{\rho}_{3}}{2 \varepsilon_{3}^{2}}\right) s_{3}^{2}-\left(\bar{\lambda}_{4}+\frac{\bar{\rho}_{4}}{2 \varepsilon_{4}^{2}}\right) s_{4}^{2} \\
& -\sum_{i=2}^{4} \frac{\sigma_{i}}{2 \kappa_{i}} \widetilde{\lambda}_{i}^{2}-\sum_{j=3,4} \frac{\xi_{j}}{2 \gamma_{j}} \widetilde{\rho}_{j}^{2}+\sum_{i=2}^{4} \frac{\sigma_{i}}{2 \kappa_{i}} \bar{\lambda}_{i}^{2}+\sum_{j=3,4} \frac{\xi_{j}}{2 \gamma_{j}} \bar{\rho}_{j}^{2} \\
& +\frac{1}{2 v_{2}} s_{2}^{2}+\frac{\chi_{3}^{2}}{2 \varepsilon_{3}^{2}} s_{3}^{2}+\frac{\chi_{4}^{2}}{2 \varepsilon_{4}^{2}} s_{4}^{2}+\frac{\nu_{2}}{2} e_{2}^{2}+\frac{\varepsilon_{3}^{2}}{2}+\frac{\varepsilon_{4}^{2}}{2}
\end{aligned}
$$

Applying $\quad|x|^{\mu}|y|^{\theta} \leq(\mu / \mu+\theta)|x|^{\mu+\theta}+(\theta / \mu+\theta) \iota^{-(\mu / \theta)}$ $|y|^{\mu+\theta}$ and taking $x=s_{i}^{2}, \quad y=1, \quad \mu=(\beta+1 / 2), \theta=(1-$ $\beta / 2)$, and $\iota=(2 / \beta+1)$ into account, one has

$$
\left|s_{i}^{2}\right|^{(\beta+1 / 2)} \leq \frac{\beta+1}{2}\left|s_{i}^{2}\right|+\frac{1-\beta}{2}\left(\frac{\beta+1}{2}\right)^{(1+\beta / 1-\beta)} .
$$

Similarly, one can obtain that

Substituting (59) into (58) gives

$$
\begin{gathered}
\left(\sum_{i=2}^{4} \frac{\sigma_{i}}{2 \kappa_{i}} \widetilde{\lambda}_{i}^{2}\right)^{(\beta+1 / 2)} \leq \frac{\beta+1}{2}\left(\sum_{i=2}^{4} \frac{\sigma_{i}}{2 \kappa_{i}} \widetilde{\lambda}_{i}^{2}\right)+\frac{1-\beta}{2}\left(\frac{\beta+1}{2}\right)^{(1+\beta / 1-\beta)}, \\
\left(\sum_{j=3,4} \frac{\xi_{j}}{2 \gamma_{j}} \tilde{\rho}_{j}^{2}\right)^{(\beta+1 / 2)} \leq \frac{\beta+1}{2}\left(\sum_{j=3,4} \frac{\xi_{j}}{2 \gamma_{j}} \widetilde{\rho}_{j}^{2}\right)+\frac{1-\beta}{2}\left(\frac{\beta+1}{2}\right)^{(1+\beta / 1-\beta)} .
\end{gathered}
$$

Substituting equations (61)-(63) into (60) yields

$$
\begin{aligned}
\dot{V}_{1} \leq & -\sum_{i=2}^{4} k_{i}\left|s_{i}^{2}\right|^{(\beta+1 / 2)}-\bar{\lambda}_{2}\left(\frac{2}{\beta+1}\left|s_{2}^{2}\right|^{(\beta+1 / 2)}-\iota\right)-\left(\bar{\lambda}_{3}+\frac{\bar{\rho}_{3}}{2 \varepsilon_{3}^{2}}\right)\left(\frac{2}{\beta+1}\left|s_{3}^{2}\right|^{(\beta+1 / 2)}-\iota\right) \\
& -\left(\bar{\lambda}_{4}+\frac{\bar{\rho}_{4}}{2 \varepsilon_{4}^{2}}\right)\left(\frac{2}{\beta+1}\left|s_{4}^{2}\right|^{(\beta+1 / 2)}-\iota\right)-\left(\frac{2}{\beta+1}\left(\sum_{i=2}^{4} \frac{\sigma_{i}}{2 \kappa_{i}} \widetilde{\lambda}_{i}^{2}\right)^{(\beta+1 / 2)}-\iota\right) \\
& -\left(\frac{2}{\beta+1}\left(\sum_{j=3,4} \frac{\xi_{j}}{2 \gamma_{j}} \tilde{\rho}_{j}^{2}\right)^{(\beta+1 / 2)}-\iota\right)+\sum_{i=2}^{4} \frac{\sigma_{i}}{2 \kappa_{i}} \bar{\lambda}_{i}^{2}+\sum_{j=3,4} \frac{\xi_{j}}{2 \gamma_{j}} \bar{\rho}_{j}^{2} \\
& +\frac{1}{2 v_{2}} s_{2}^{2}+\frac{\chi_{3}^{2}}{2 \varepsilon_{3}^{2}} s_{3}^{2}+\frac{\chi_{4}^{2}}{2 \varepsilon_{4}^{2}} s_{4}^{2}+\frac{\nu_{2}}{2} e_{2}^{2}+\frac{\varepsilon_{3}^{2}}{2}+\frac{\varepsilon_{4}^{2}}{2}
\end{aligned}
$$


where $\iota=(1-\beta / 2)(\beta+1 / 2)^{(2 \beta / 1-\beta)}>0$.

With Lemma 3, (64) becomes

$$
\begin{aligned}
\dot{V}_{1} \leq & -\left(k_{2}+\frac{2 \bar{\lambda}_{2}}{\beta+1}\right) 2^{(\beta+1 / 2)}\left|\frac{1}{2} s_{2}^{2}\right|^{(\beta+1 / 2)}-\left(k_{3}+\frac{2}{\beta+1}\left(\bar{\lambda}_{3}+\frac{\bar{\rho}_{3}}{2 \varepsilon_{3}^{2}}\right)\right) 2^{(\beta+1 / 2)}\left|\frac{1}{2} s_{3}^{2}\right|^{(\beta+1 / 2)} \\
& -\left(k_{4}+\frac{2}{\beta+1}\left(\bar{\lambda}_{4}+\frac{\bar{\rho}_{4}}{2 \varepsilon_{4}^{2}}\right)\right) 2^{(\beta+1 / 2)}\left|\frac{1}{2} s_{4}^{2}\right|^{(\beta+1 / 2)}-\frac{2}{\beta+1} \sigma_{0}^{(\beta+1 / 2)}\left(\frac{1}{2} \sum_{i=2}^{4} \frac{1}{\kappa_{i}} \widetilde{\lambda}_{i}^{2}\right)^{(\beta+1 / 2)} \\
& -\frac{2}{\beta+1} \xi_{0}^{(\beta+1 / 2)}\left(\frac{1}{2} \sum_{j=3,4} \frac{1}{\gamma_{j}} \widetilde{\rho}_{j}^{2}\right)^{(\beta+1 / 2)}+\iota\left(\bar{\lambda}_{2}+\bar{\lambda}_{3}+\frac{\bar{\rho}_{3}}{2 \varepsilon_{3}^{2}}+\bar{\lambda}_{4}+\frac{\bar{\rho}_{4}}{2 \varepsilon_{4}^{2}}+2\right) \\
& +\sum_{i=2}^{4} \frac{\sigma_{i}}{2 \kappa_{i}} \bar{\lambda}_{i}^{2}+\sum_{j=3,4} \frac{\xi_{j}}{2 \gamma_{j}} \bar{\rho}_{j}^{2}+\frac{1}{2 v_{2}} s_{2}^{2}+\frac{\chi_{3}^{2}}{2 \varepsilon_{3}^{2}} s_{3}^{2}+\frac{\chi_{4}^{2}}{2 \varepsilon_{4}^{2}} s_{4}^{2}+\frac{\nu_{2}}{2} e_{2}^{2}+\frac{\varepsilon_{3}^{2}}{2}+\frac{\varepsilon_{4}^{2}}{2}
\end{aligned}
$$

where $\sigma_{0}=\min \left(\sigma_{i}\right)>0$ and $\xi_{0}=\min \left(\xi_{j}\right)>0$.

By choosing appropriate parameters, following conditions will hold:

$$
\left\{\begin{array}{l}
\Psi_{1}=\min \left(\left(k_{2}+\frac{2 \bar{\lambda}_{2}}{\beta+1}\right) 2^{(\beta+1 / 2)},\left(k_{3}+\frac{2}{\beta+1}\left(\bar{\lambda}_{3}+\frac{\bar{\rho}_{3}}{2 \varepsilon_{3}^{2}}\right)\right) 2^{(\beta+1 / 2)},\left(k_{4}+\frac{2}{\beta+1}\left(\bar{\lambda}_{4}+\frac{\bar{\rho}_{4}}{2 \varepsilon_{4}^{2}}\right)\right) 2^{(\beta+1 / 2)}\right)>0, \\
\Psi_{2}=\frac{2}{\beta+1} \sigma_{0}^{(\beta+1 / 2)}>0, \\
\Psi_{3}=\frac{2}{\beta+1} \xi_{0}^{(\beta+1 / 2)}>0, \\
\Psi_{0}=\iota\left(\bar{\lambda}_{2}+\bar{\lambda}_{3}+\frac{\bar{\rho}_{3}}{2 \varepsilon_{3}^{2}}+\bar{\lambda}_{4}+\frac{\bar{\rho}_{4}}{2 \varepsilon_{4}^{2}}+2\right)+\sum_{i=2}^{4} \frac{\sigma_{i}}{2 \kappa_{i}} \bar{\lambda}_{i}^{2}+\sum_{j=3,4} \frac{\xi_{j}}{2 \gamma_{j}} \bar{\rho}_{j}^{2}+\frac{1}{2 \gamma_{2}} s_{2}^{2}+\frac{\chi_{3}^{2}}{2 \varepsilon_{3}^{2}} s_{3}^{2}+\frac{\chi_{4}^{2}}{2 \varepsilon_{4}^{2}} s_{4}^{2}+\frac{\nu_{2}}{2} e_{2}^{2}+\frac{\varepsilon_{3}^{2}}{2}+\frac{\varepsilon_{4}^{2}}{2}>0 .
\end{array}\right.
$$

Then, (65) can be rewritten as

$$
\begin{aligned}
\dot{V}_{1} & \leq-\Psi_{1}\left(\frac{1}{2} \sum_{i=2}^{4}\left(s_{i}^{2}\right)\right)^{(\beta+1 / 2)}-\Psi_{2}\left(\frac{1}{2} \sum_{i=2}^{4} \frac{1}{\kappa_{i}} \tilde{\lambda}_{i}^{2}\right)^{(\beta+1 / 2)}-\Psi_{3}\left(\frac{1}{2} \sum_{i=3}^{4} \frac{1}{\gamma_{i}} \tilde{\rho}_{i}^{2}\right)^{(\beta+1 / 2)}+\Psi_{0} \\
& \leq-\Psi V_{1}^{(\beta+1 / 2)}
\end{aligned}
$$

where $\Psi=\min \left(\Psi_{1}, \Psi_{2}, \Psi_{3}\right)>0$.

According to Lemma 1 , the system will converge to the equilibrium point in finite time and the settling time is given by

$$
t_{r} \leq \frac{V_{1}^{1-\beta}\left(x_{0}\right)}{\Psi(1-\beta)} .
$$

Furthermore, all the state in the closed-loop guidance system are uniformly ultimately bounded.

Then, consider another Lyapunov function:

$$
V_{s}=\sum_{i=2}^{4} \frac{1}{2} s_{i}^{2} .
$$


With (34), (40), and (47), the time derivative of $V_{s}$ becomes

$$
\begin{aligned}
\dot{V}_{s} & =\sum_{i=2}^{4} s_{i} \dot{s}_{i} \\
& \leq-\sum_{i=2}^{4} k_{i}\left|s_{i}\right|^{\beta+1}-\sum_{i=2}^{4} \hat{\lambda}_{i} s_{i}^{2}+s_{2} e_{2} .
\end{aligned}
$$

Since $s_{2} e_{2} \leq\left(1 / 2 v_{2}\right) s_{2}^{2}+\left(\nu_{2} / 2\right) e_{2}^{2}$ with $v_{2}$ is a positive constant, we have

$$
\begin{aligned}
\dot{V}_{s} & \leq-\sum_{i=2}^{4} k_{i}\left|s_{i}\right|^{\beta+1}-\sum_{i=2}^{4} \widehat{\lambda}_{i} s_{i}^{2}+\frac{1}{2 \nu_{2}} s_{2}^{2}+\frac{\nu_{2}}{2} e_{2}^{2} \\
& \leq-\sum_{i=2}^{4} k_{i}\left|s_{i}\right|^{\beta+1}-\left(\hat{\lambda}_{2}-\frac{1}{2 \nu_{2}}\right) s_{2}^{2}-\hat{\lambda}_{3} s_{3}^{2}-\hat{\lambda}_{4} s_{4}^{2}+\frac{\nu_{2}}{2} e_{2}^{2} \\
& \leq-2^{(\beta+1 / 2)} \min \left(k_{2}, k_{3}, k_{4}\right) \sum_{i=2}^{4}\left(\frac{s_{i}^{2}}{2}\right)^{(\beta+1 / 2)}-2 \min \left(\hat{\lambda}_{2}-\frac{1}{2 \nu_{2}}, \hat{\lambda}_{3}, \hat{\lambda}_{4}\right) \sum_{i=2}^{4}\left(\frac{s_{i}^{2}}{2}\right)+\frac{\nu_{2}}{2} e_{2}^{2}
\end{aligned}
$$

From (71), if we choose appropriate adaptive laws for $\hat{\lambda}_{i}(i=2,3$, and 4$)$ and large enough $\nu_{2}$ to guarantee $\widehat{\lambda}_{2}-\left(1 / 2 v_{2}\right)>0, \widehat{\lambda}_{3}>0$, and $\widehat{\lambda}_{4}>0$, one can obtain that

$$
\dot{V}_{s} \leq-\Gamma_{1} V_{s}^{(\beta+1 / 2)}-\Gamma_{2} V_{s}+\Gamma_{0} \leq-\Gamma_{1} V_{s}^{(\beta+1 / 2)}-\Gamma_{2} V,
$$

where $\quad \Gamma_{1}=2^{(1+\beta / 2)} \min \left(k_{i}\right)(i=2,3,4)>0$ and $\Gamma_{2}=2 \min \left(\widehat{\lambda}_{2}-\left(1 / 2 v_{2}\right), \hat{\lambda}_{3}, \widehat{\lambda}_{4}\right)>0$.

Thus, according to Lemma $2, s_{i}$ will converge to zero in finite time bounded by

$$
t_{s} \leq \frac{2}{\Gamma_{2}(1-\beta)} \ln \frac{\Gamma_{2} V_{s}(0)^{(1-\beta / 2)}+\Gamma_{1}}{\Gamma_{1}} .
$$

When $s_{2}=0$ and $\dot{s}_{2}=0$ are achieved, the following equation can be obtained:

$$
\dot{s}_{2}=\dot{x}_{2}+\lambda_{11} \operatorname{sig}\left(x_{1}\right)^{\alpha_{1}}+\lambda_{12} \operatorname{sig}\left(x_{2}\right)^{\alpha_{2}}=0 .
$$

Namely,

$$
\dot{x}_{2}=-\lambda_{11} \operatorname{sig}\left(x_{1}\right)^{\alpha_{1}}-\lambda_{12} \operatorname{sig}\left(x_{2}\right)^{\alpha_{2}} .
$$

Since $\dot{x}_{1}=x_{2},(75)$ can be rewritten as

$$
\ddot{x}_{1}+\lambda_{12} \operatorname{sig}\left(\dot{x}_{1}\right)^{\alpha_{2}}+\lambda_{11} \operatorname{sig}\left(x_{1}\right)^{\alpha_{1}}=0 .
$$

According to Lemma 4, once parameters $\lambda_{12}$ and $\lambda_{11}$ are selected to make polynomial $p^{2}+\lambda_{12} p^{\alpha_{2}}+\lambda_{1} p^{\alpha_{1}}$ Hurwitz, $x_{1}$ and $x_{2}$ can converge to the equilibrium point in finite time which can be calculated as

$$
t_{x} \leq \frac{\left(3+\alpha_{1}\right) V_{0}^{\left(1-\alpha_{1}\right) /\left(3+\alpha_{1}\right)}\left(x_{1}(0), x_{2}(0)\right)}{K\left(1-\alpha_{1}\right)},
$$

where $K$ is a positive constant.

Therefore, the designed composite guidance law can guarantee the finite-time stability of the closed-loop system and drive the sliding modes $s_{i}$ and states $x_{1}$ and $x_{2}$ to converge to zero in finite time with the total settling time $t_{c}=t_{o}+t_{r}+t_{s}+t_{x}$.

The proof of Theorem 2 has been completed.

Remark 7. In most existing literature, the "differential explosion problem" of the traditional backstepping method is solved by introducing the first-order filter or tracking differentiator. In this study, the proposed adaptive laws are applied to estimate the derivative of virtual control inputs.

Remark 8. For the global guidance system, $\lambda_{11}$, $\lambda_{12}, \alpha_{1}, \beta_{1}, \kappa_{i}, \sigma_{i}, \varepsilon_{i}, \gamma_{j}, \varepsilon_{j}$, and $\xi_{j}(i=2,3$, and $4 ; j=3$ and 4$)$ are all positive parameters to be regulated. Specifically, $k_{i}$ can be selected large enough to stabilize each subsystem. For subsystem $x_{2}, \kappa_{2}$ is selected large enough and $\sigma_{2}$ is selected small enough, while for the subsystems $x_{3}$ and $x_{4}$, regulation laws of $\kappa_{3}, \kappa_{4}$ and $\sigma_{3}, \sigma_{4}$ are the opposite. $\gamma_{j}$ and $\xi_{j}$ can be selected small enough, while $\varepsilon_{i}$ can be selected large enough. With the satisfactory parameters, the convergence characteristic of the closed-loop system can be guaranteed. In other words, the state errors can converge to any small interval of the origin. Besides, when the system gets convergent, $\lambda_{11}, \lambda_{12}, \alpha_{1}$, and $\alpha_{2}$ will further balance the LOS error and miss distance. As $\lambda_{11}$ and $\lambda_{12}$ increase, the miss distance will increase and the LOS error decreases. Besides, oversized $\lambda_{11}$ and $\lambda_{12}$ will result in the failure of the mission. Thus, appropriate small $\lambda_{11}$ and $\lambda_{12}$ should be determined. The parameters $\alpha_{1}$ and $\beta$ mainly affect the convergence rate. Large $\alpha_{1}$ and $\beta$ will lead to the oscillation of the transient process, while small $\alpha_{1}$ and $\beta$ will degrade the convergence speed and bring about the interception failure. Therefore, when tuning these parameters, selecting proper $\lambda_{11}, \lambda_{12}, \alpha_{1}$, and $\beta$ first so as to guarantee the relatively fast convergence rate with acceptable miss distance and LOS error. Then, choosing appropriate $k_{i}, \kappa_{i}, \sigma_{i}, \varepsilon_{i}, \gamma_{j}, \varepsilon_{j}$, and $\xi_{j}$ to stabilize 
each subsystem. Finally, these parameters can be fine-tuned to improve comprehensive performance.

To further explain the tuning process, the corresponding main tuning steps can be described as Table 1 .

\section{Simulation Results and Discussions}

Numerical examples are performed in this section to verify the effectiveness of the proposed composite guidance law. The simulation conditions are listed in Table 2. The maximal saturation acceleration of the interceptor missile is $40 \mathrm{~g}$ $(g=9.8)$.

We assume that the relative disturbance $r$, the LOS angle $\theta$, and their rates ( $\dot{r}$ and $\dot{\theta}$ ) can be provided by the seeker. The interception performance is evaluated by the interception time, miss distance, LOS error, and control energy (denoted as $\left.\int a_{m c}^{2} \mathrm{~d} \tau\right)$. The designed parameters for the proposed guidance scheme (denoted as AISMB-FTDO) are chosen as $\lambda_{11}=0.1, \lambda_{12}=0.05, \alpha_{1}=\alpha=4 / 5, \beta=4 / 5, \quad k_{2}=6, \kappa_{2}=10$, $\sigma_{2}=0.1, \delta_{2}=0.005, k_{3}=0.5, \kappa_{3}=0.00001, \sigma_{3}=1000, \delta_{3}=$ $1000, \varepsilon_{3}=100, \gamma_{3}=0.0001, \xi_{3}=0.1, k_{4}=0.5, \kappa_{4}=0.0001$, $\sigma_{4}=1000, \delta_{4}=4000, \varepsilon_{4}=1000, \gamma_{4}=0.01, \xi_{4}=0.01, k_{21}=$ $10, k_{22}=5, k_{23}=4, p=0.6, q=1.4$, and $\omega_{2}=\omega_{3}=\omega_{4}=$ 0.00001 .

To make a better showcase, the terminal sliding mode backstepping (SMB) guidance law [22] with the ESO [10] (denoted as SMB-ESO) and the finite time convergent (FTC) guidance law with SMO [31] (denoted as FTC-SMO) are also introduced into the simulation for comparison.

The SMB-ESO is defined as

$$
\begin{aligned}
& \mathrm{SMB}:\left\{\begin{array}{l}
s_{2}=x_{2}+a_{1} x_{1} \operatorname{sig}\left(x_{1}\right)^{p_{1}}, \\
x_{3 d}=g_{2}^{-1}\left(-f_{2}-\alpha_{1}\left|x_{1}\right|^{p-1} x_{2}-\lambda_{21} s_{2}-\lambda_{22} \operatorname{sig}\left(s_{2}\right)^{p_{2}}+z_{22}\right), \\
\tau \dot{x}_{3 c}+x_{3 c}=x_{3 d}, \\
x_{3 c}(0)=x_{3 d}(0), \\
s_{3}=x_{3}-x_{3 c}, \\
x_{4 d}=-\lambda_{31} \operatorname{sig}\left(s_{3}\right)^{p_{3}}-\lambda_{32} s_{3}-g_{2} s_{2}-\dot{x}_{3 c}, \\
\tau \dot{x}_{4 c}+x_{4 c}=x_{4 d}, \\
x_{4 c}(0)=x_{4 d}(0), \\
s_{4}=x_{4}-x_{4 c}, \\
u=g_{4}^{-1}\left(-f_{3}-s_{3}-\lambda_{41} \operatorname{sig}\left(s_{4}\right)^{p_{4}}-\lambda_{42} s_{4}-\dot{x}_{4 c}+z_{42}\right),
\end{array}\right. \\
& \text { ESO: }\left\{\begin{array}{l}
e_{21}=z_{21}-x_{2}, \\
\dot{z}_{21}=f_{2}+g_{2} x_{3 d}-\beta_{21} e_{21}+z_{22}, \\
\dot{z}_{22}=-\beta_{22} \text { fal }\left(e_{21}, \alpha_{2}, \delta_{2}\right)
\end{array}\right.
\end{aligned}
$$

where $\mathrm{fal}(\cdot)$ is a nonlinear function defined as

$$
\operatorname{fal}(e, \alpha, \delta)= \begin{cases}\operatorname{sig}(e)^{\alpha}, & |e|>\delta \\ \frac{e}{\delta^{1-\alpha}}, & \text { otherwise }\end{cases}
$$

with $\delta$ is a positive constant. The parameters of SMB-ESO can be chosen as $a_{1}=0.9, p_{1}=0.9, \lambda_{21}=10, \lambda_{22}=2, p_{2}=$ $1.15, \tau=0.02, \lambda_{31}=0.01, \lambda_{32}=0.3, p_{3}=1.5, p_{4}=1.5, \lambda_{41}=$
$0.9, \lambda_{42}=0.5, \beta_{21}=10, \beta_{22}=100, \alpha_{2}=0.91$, and $\delta_{2}=$ 0.00001 .

The FTC-SMO is defined as

$$
\text { FTC: } u=-N_{1} \dot{r} \dot{q}+N_{2} \operatorname{sig}(\dot{q})^{q}+z_{1},
$$

$$
\mathrm{SMO}:\left\{\begin{array}{l}
\dot{z}_{0}=-2 \frac{\dot{r}}{r} z_{0}+g_{2} a_{m c}+v_{0}, \\
v_{0}=-l_{2} L^{1 / 3} \operatorname{sig}\left(e_{21}\right)^{2 / 3}+z_{1} \\
e_{21}=z_{0}-x_{2}, \\
\dot{z}_{1}=v_{1}, \\
v_{1}=-l_{1} L^{1 / 2} \operatorname{sig}\left(z_{1}-v_{0}\right)^{2 / 3}+z_{2} \\
\dot{z}_{2}=l_{0} L \operatorname{sign}\left(z_{2}-v_{1}\right) .
\end{array}\right.
$$

The parameters of FTC-SMO are selected as $N_{1}=4, N_{2}=30, q=0.5, l_{2}=2, l_{1}=1.5, l_{0}=1.1$, and $L=$ 400 .

Remark 9. To alleviate the chattering phenomenon, the sign function $\operatorname{sign}(x)$ is replaced by the following continuous function:

$$
\tanh \left(\frac{x}{\eta}\right)=\frac{e^{x / \eta}-e^{-x / \eta}}{e^{x / \eta}+e^{-x / \eta}},
$$

where $\eta>0$ is a sufficient small constant.

In order to illustrate the effectiveness of the proposed guidance law, the simulation examples are divided into two kinds of target maneuverings, constant-maneuvering and cosine maneuvering. Detailed simulation results and relevant discussions are shown as below.

4.1. Scenario 1. In this scenario, the simulation results for the constant-maneuvering target are presented, in which the target acceleration is chosen as $a_{t}=10 \mathrm{~g}$. The corresponding simulation curves of this scenario are shown in Figures 3-9. The trajectories of the missile and target are shown in Figure 3, which can reveal that these three guidance laws could guarantee the successful interception. Besides, it is obvious that the curves of AISMB-FTDO and FTC-SMO are smoother than that of SMB-ESO. Nevertheless, the trajectory of AISMB-FTDO is flatter and easier to implement in practical engineering, while FTC-SMO does not have this property. Figure 4 reveals that both AISMB-FTDO and SMB-ESO can achieve the certain LOS angle, while FTCSMO cannot. The reason for this result is that FTC-SMO does not consider the LOS angle constraint. The LOS rate curves are shown in Figure 5. As seen from Figures 4 and 5, although SMB-ESO can achieve the certain LOS angle, the LOS angle curve displays some chattering caused by the divergence of the LOS angular rates during the end phase. 
TABLE 1: The main parameter tuning steps.

(i) Select $\lambda_{11}$ and $\lambda_{12}$ to make the polynomial $p^{2}+\lambda_{12} p^{\alpha_{2}}+\lambda_{1} p^{\alpha_{1}}$ Hurwitz and set $\alpha_{1}$ and $\beta$ as 0.5

(ii) Fix $\lambda_{11}, \lambda_{12}, \alpha_{1}$, and $\beta$, set $\kappa_{2}, \sigma_{2}$, and $\varepsilon_{2}$ as zero, and then tune the parameter $k_{2}$ to stabilize the subsystem $x_{2}$; if this subsystem looks stable or has a stable trend, go to step iii; otherwise, continue this step

(iii) Adjust the parameter $\kappa_{2}, \sigma_{2}$, and $\varepsilon_{2}$ to further stabilize the subsystem $x_{2}$; if this subsystem looks stable or has a stable trend, go to step iv; otherwise, continue this step

(iv) Fix $\lambda_{11}, \lambda_{12}, \alpha_{1}, \beta, k_{2}, \kappa_{2}, \sigma_{2}$, and $\varepsilon_{2}$, set $\kappa_{3}, \sigma_{3}$, and $\varepsilon_{3}$ as zero, and then tune the parameters $k_{3}, \gamma_{3}, \varepsilon_{3}$, and $\xi_{3}$ to stabilize the subsystem $x_{3}$; if this subsystem looks stable or has a stable trend, go to step v; otherwise, continue this step

(v) Adjust the parameter $\kappa_{3}, \sigma_{3}$, and $\varepsilon_{3}$ to further stabilize the subsystem $x_{3}$; if this subsystem looks stable or has a stable trend, go to step vi; otherwise, continue this step

(vi) Fix $\lambda_{11}, \lambda_{12}, \alpha_{1}, \beta, k_{2}, \kappa_{2}, \sigma_{2}, \varepsilon_{2}, k_{3}, \kappa_{3}, \sigma_{3}, \varepsilon_{3}, \gamma_{3}, \varepsilon_{3}$, and $\xi_{3}$, set $\kappa_{4}, \sigma_{4}$, and $\varepsilon_{4}$ as zero, and then tune the parameters $k_{4}, \gamma_{4}, \varepsilon_{4}$, and $\xi_{4}$ to stabilize the subsystem $x_{4}$; if this subsystem looks stable or has a stable trend, go to step vii; otherwise, continue this step

(vii) Adjust the parameter $\kappa_{4}, \sigma_{4}$, and $\varepsilon_{4}$ to further stabilize the subsystem $x_{4}$; if this subsystem looks stable or has a stable trend, go to step viii; otherwise, continue this step

(viii) In this step, the stability of closed-loop is guaranteed; then, re-adjust the parameters $\lambda_{11}, \lambda_{12}, \alpha_{1}$, and $\beta$ to balance the miss distance, LOS error, and convergence speed; these parameters will be further fine-tuned so as to achieve better overall performance

TABLE 2: Initial conditions for the simulation examples.

\begin{tabular}{lc}
\hline State (unit) & Value \\
\hline$x_{m}(\mathrm{~m})$ & 0 \\
$y_{m}(\mathrm{~m})$ & 0 \\
$V_{m}(\mathrm{~m} / \mathrm{s})$ & 600 \\
$\gamma_{m}\left({ }^{\circ}\right)$ & 60 \\
$\xi$ & 0.707 \\
$\theta_{f}\left({ }^{\circ}\right)$ & 20 \\
$x_{t}(\mathrm{~m})$ & 5000 \\
$y_{t}(\mathrm{~m})$ & 2500 \\
$V_{t}(\mathrm{~m} / \mathrm{s})$ & 300 \\
$\gamma_{t}\left({ }^{\circ}\right)$ & 10 \\
$\omega_{n}$ & 10 \\
\hline
\end{tabular}

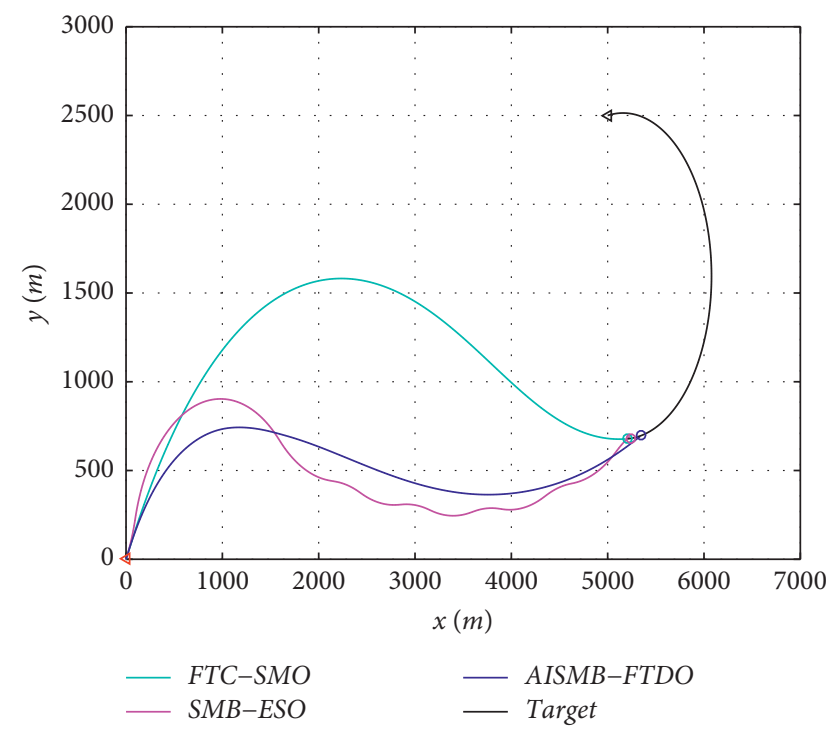

FIgURE 3: Trajectories of the missile and target in scenario 1.

Howbeit, AISMB-FTDO could ensure little LOS angle error without chattering. The actual guidance command curves are shown in Figure 6. Apparently, the control input curves of AISMB-FTDO and FTC-SMO are much smoother, while SMB-ESO exhibits serious saturation. This saturation

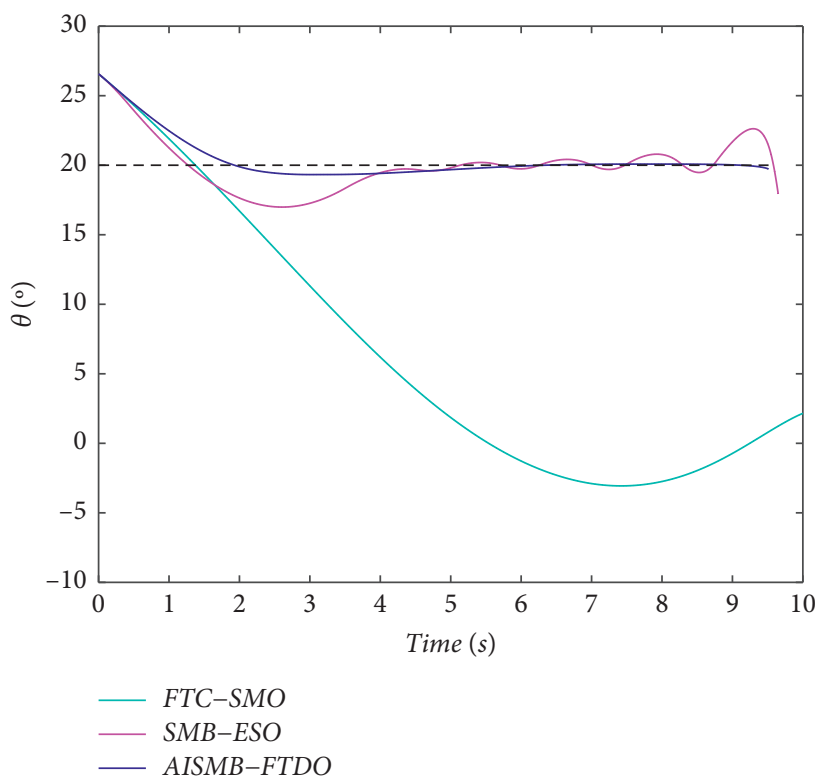

FIgURE 4: Curves of the LOS angle in scenario 1.

phenomenon will degrade the guidance performance and produce extremely large control energy. It can be seen from Figure 7 that the tracking error curves of SMO and FTDO can converge to zero in shorter time, while ESO need longer time. However, it is obvious that the curve of SMO causes a serious chattering phenomenon, while FTDO does not have this problem. This is because the sign function in FTDO is hidden in the integral term and the continuity of estimation value can be guaranteed. Therefore, this result further indicates that FTDO has better disturbance observation ability. The adaptive gains and adaptive parameters are shown in Figures 8 and 9, respectively. Both of these parameters can vary with respect to time and converge to certain values finally. These curves can further confirm the effectiveness of the designed adaptive laws. The interception results obtained from the guidance laws are summarized in Table 3. Compared with FTC-SMO and SMB-ESO, AISMB-FTDO possesses less interception time, smaller miss distance, less LOS 


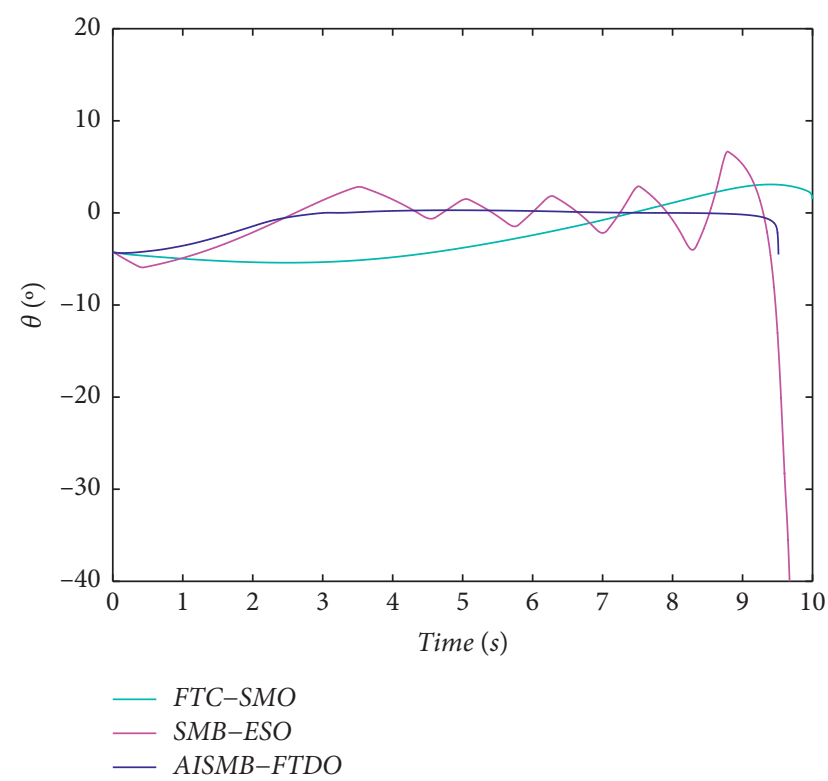

FIgURE 5: Curves of the LOS angular rate in scenario 1.

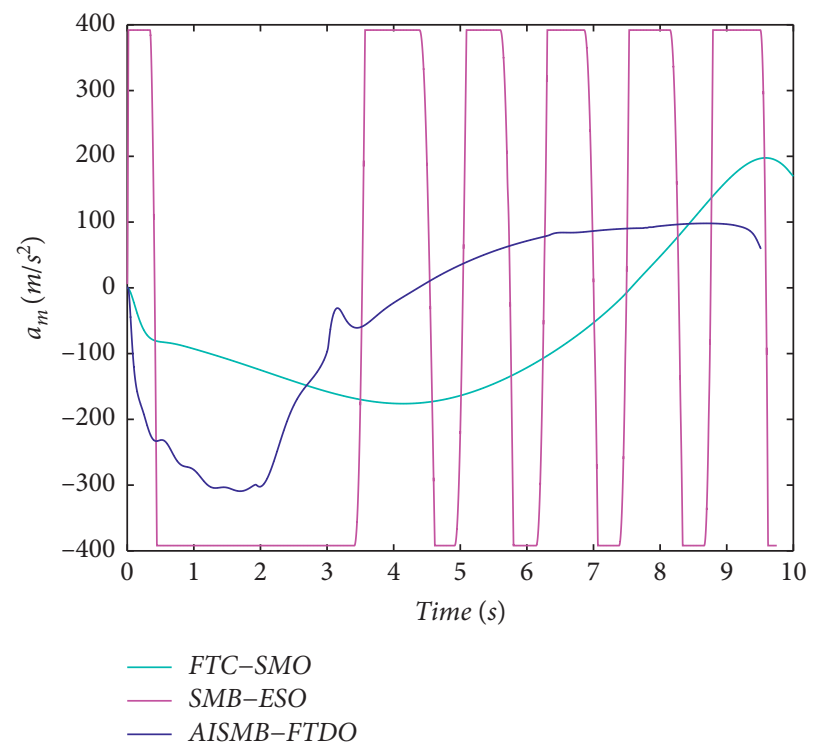

FIgURE 6: Curves of missiles' actual accelerations in scenario 1.

error, and a relatively small control energy. It is worth noting that although FTC-SMO exhibits the largest LOS error, the required control energy is the smallest. The reason for this phenomenon is that FTC-SMO does not consider the LOS angle constraint. Besides, compared with SMB-ESO, the control energy of AISMB-FTDO is much smaller since the gain regulating laws work well during the interception process. Thus, we can draw the conclusion that AISMBFTDO has better comprehensive performance than FTCSMO and SMB-ESO.

4.2. Scenario 2. To further verify the capability of the proposed AISMB-FTDO guidance law in a more complicated situation, the target acceleration is described as

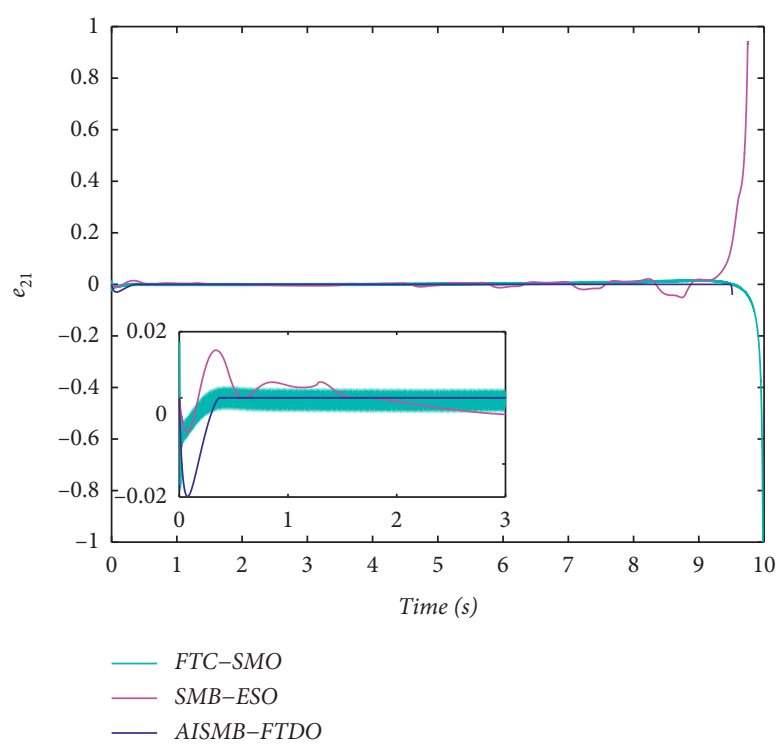

Figure 7: Curves of observation errors from the disturbance observer in scenario 1.

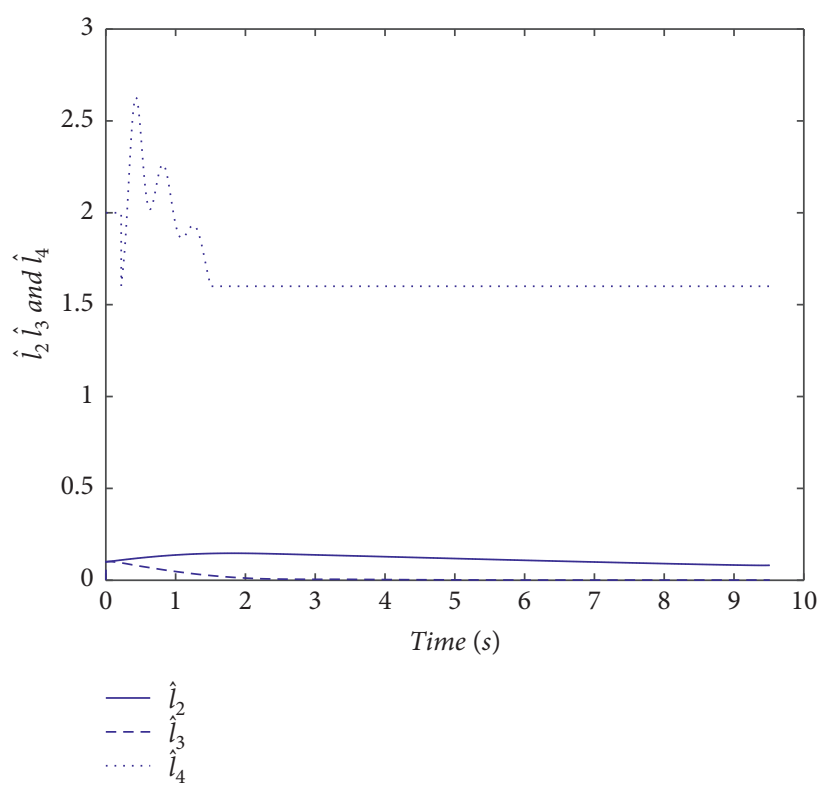

FIgURE 8: Curves of adaptive gains $\widehat{\lambda}_{i}(i=2,3$, and 4) in scenario 1 .

$a_{t}=10 \mathrm{~g} \cos (\pi t / 8)$ in this scenario. The design requirement and the guidance parameters are unchanged. It is shown from Table 4 that the AISMB-FTDO guidance law still possesses less interception time, smaller miss disturbance, less LOS error, and relatively small control energy than FTCSMO and SMB-ESO. Figure 10 depicts the trajectories of the missile and target in scenario 2. It is obvious that the trajectories of AISMB-FTDO and FTC-SMO can still be smooth, while SMB-ESO exhibits slow chattering. The LOS angle and LOS angular rate are shown in Figures 11 and 12, respectively. The results in these two figures present that the proposed AISMB-FTDO guidance law can still guarantee the accuracy of the LOS angle, whereas FTC-SMO and SMB- 


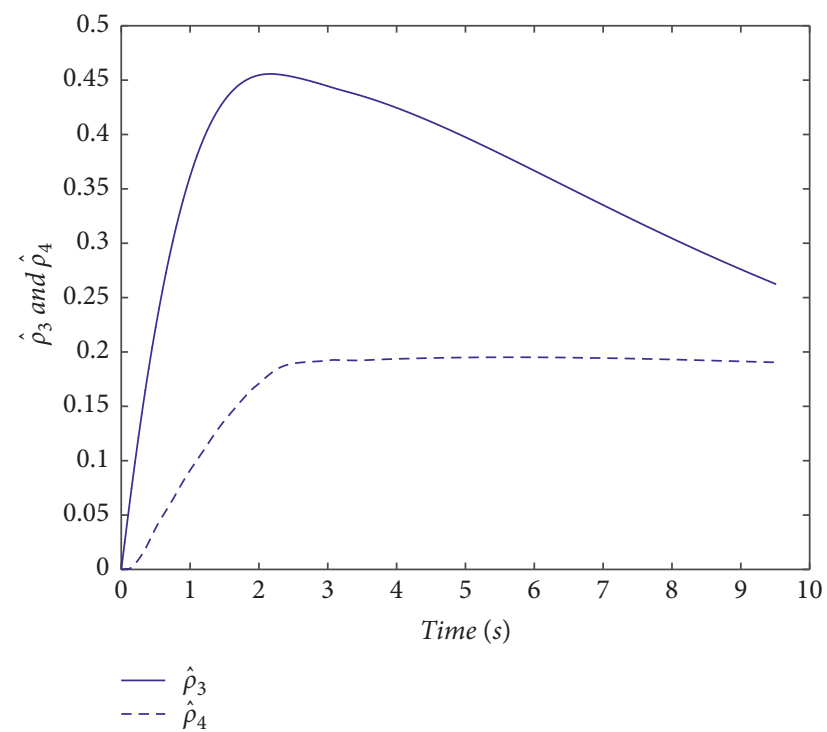

Figure 9: Curves of adaptive parameters $\widehat{\rho}_{j}(j=3$ and 4$)$ in scenario 1 .

TABLE 3: Interception result data of scenario 1.

\begin{tabular}{lcccc}
\hline Guidance law & Interception time $(\mathrm{s})$ & Miss distance $(\mathrm{m})$ & LOS error $\left(^{\circ}\right)$ & ${\text { Control energy }\left(\mathrm{m}^{2} / \mathrm{s}^{4}\right)}^{\circ}$ \\
\hline FTC-SMO & 10.001 & 0.4336 & -17.8479 & $1.7734 \times 10^{5}$ \\
SMB-ESO & 9.866 & 0.3968 & -2.0710 & $2.5870 \times 10^{8}$ \\
AISMB-FTDO & 9.514 & 0.2763 & -0.2937 & $2.2498 \times 10^{5}$ \\
\hline
\end{tabular}

TABLE 4: Interception result data of scenario 2.

\begin{tabular}{lcccc}
\hline Guidance law & Interception time $(\mathrm{s})$ & Miss distance $(\mathrm{m})$ & LOS error $\left(^{\circ}\right)$ & ${\text { Control energy }\left(\mathrm{m}^{2} / \mathrm{s}^{4}\right)}^{\circ}$ \\
\hline FTC-SMO & 18.857 & 0.1321 & -8.4666 & $1.2877 \times 10^{5}$ \\
SMB-ESO & 17.939 & 0.9362 & 6.9654 & $4.6918 \times 10^{7}$ \\
AISMB-FTDO & 17.675 & 0.1921 & -0.0579 & $1.7983 \times 10^{5}$ \\
\hline
\end{tabular}

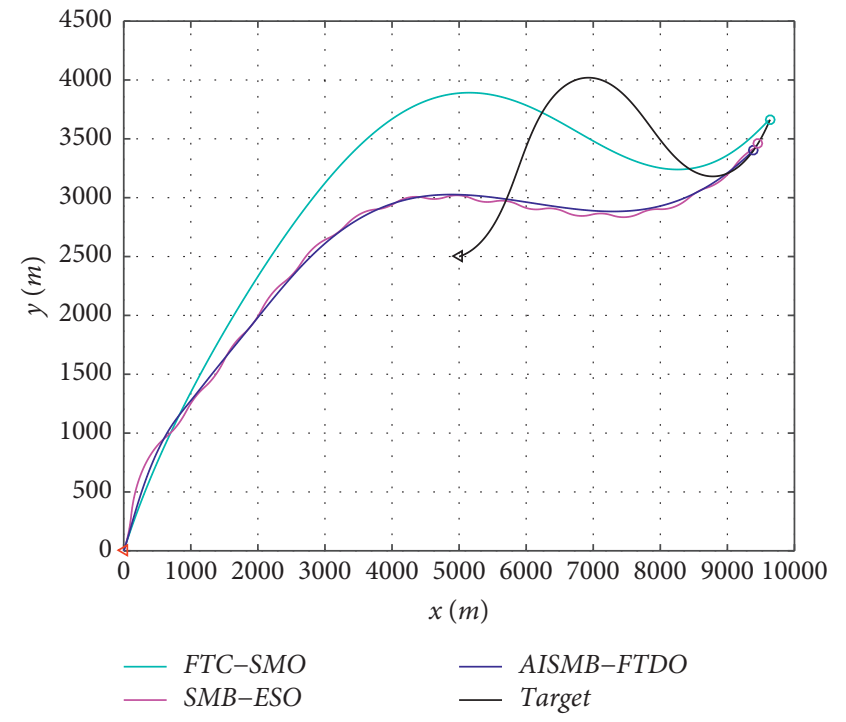

Figure 10: Trajectories of the missile and target in scenario 2.

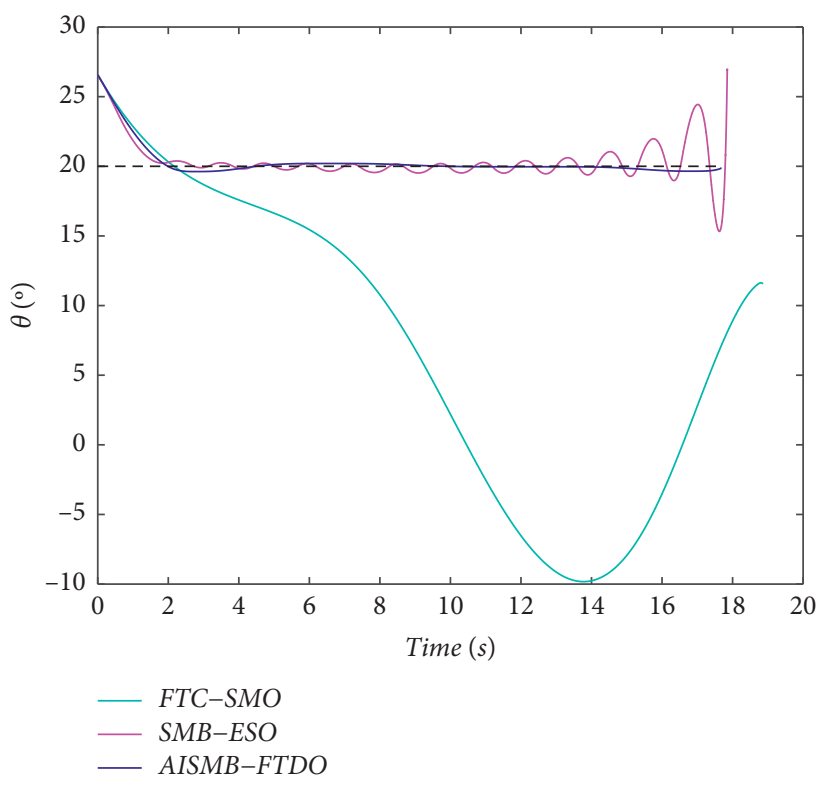

Figure 11: Curves of the LOS angle in scenario 2. 


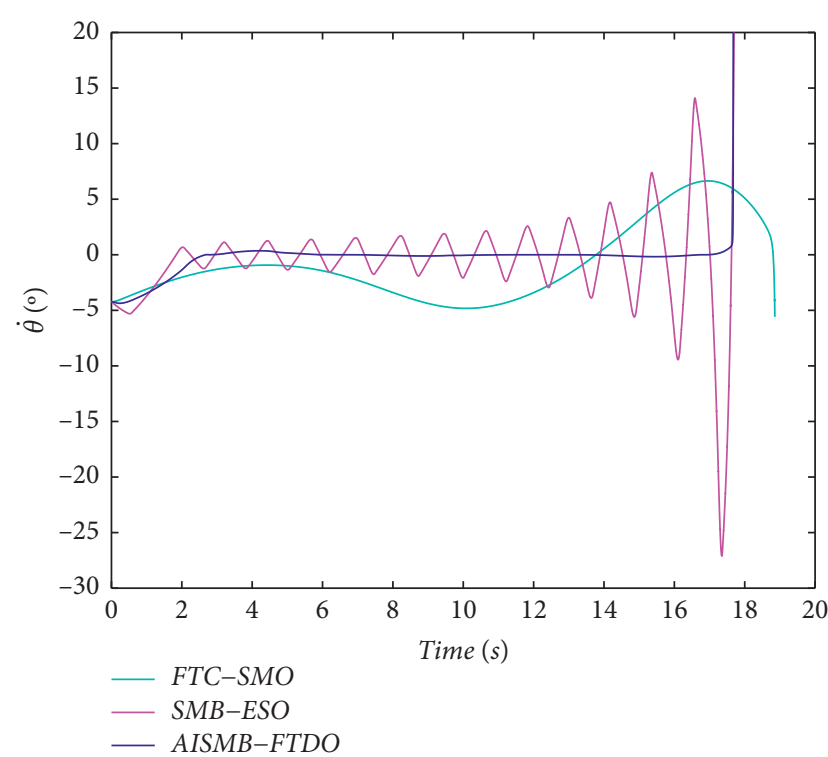

FIgURE 12: Curves of the LOS angular rate in scenario 2.

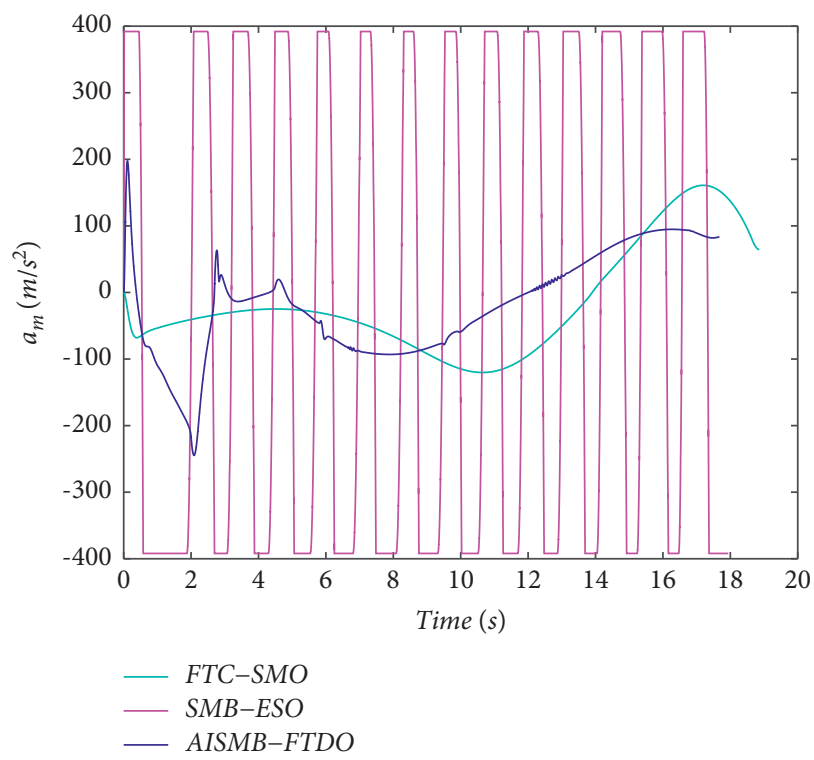

FIgURE 13: Curves of missiles' actual acceleration in scenario 2.

ESO have worse performance. Figure 13 exhibits the accelerations of the missiles. It can be concluded that the curves of AISMB-FTDO and FTC-SMO are smoother. Besides, the curve of SMB-ESO is pretty difficult for the actual implement. Figure 14 shows that the estimation accuracy of FTDO is still favorable in the presence of high maneuverability, while SMO still causes severe chattering problem. The adaptive gains and adaptive parameters are shown in Figures 15 and 16. These variables determine the satisfactory guidance performance.

Remark 10. The observation errors in Figures 7 and 14 both show large sudden changes at the ending of the interception, which will degrade the actual control effect. This may result

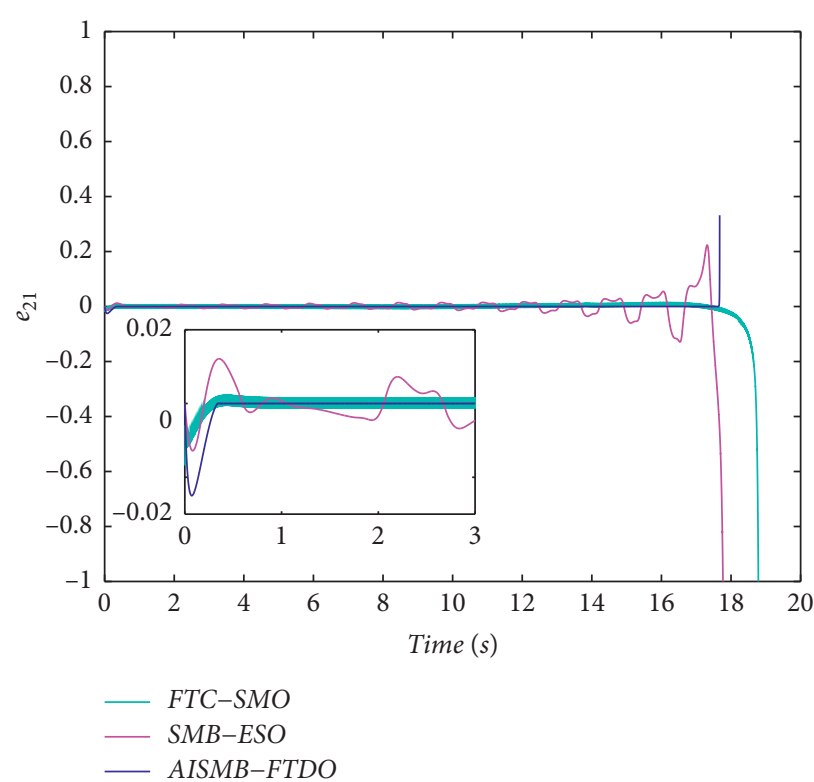

FIgURE 14: Curves of observation errors from the disturbance observer in scenario 2 .

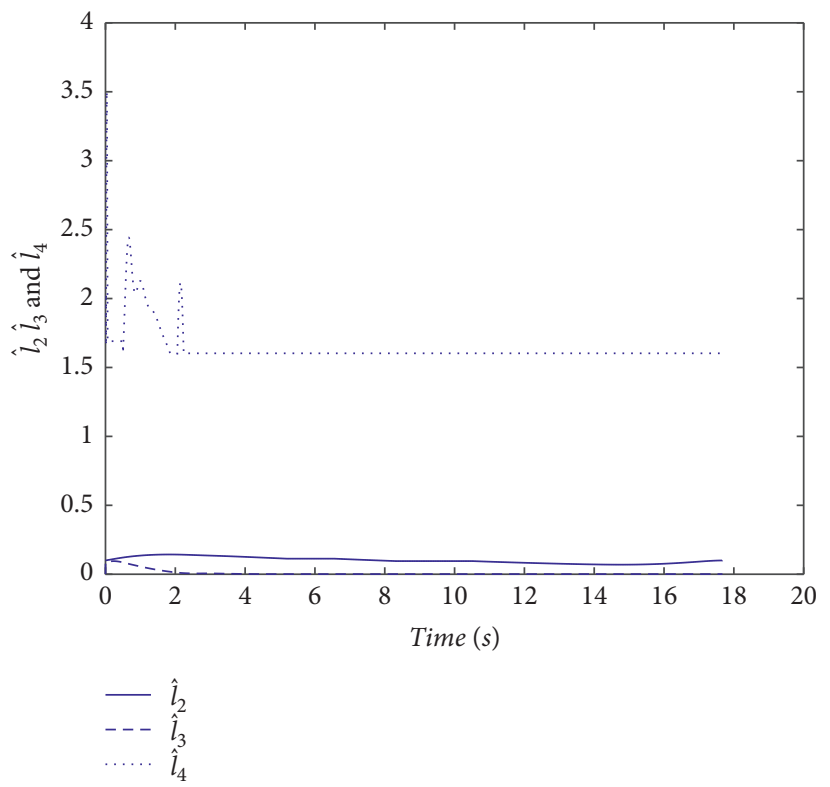

FIgURE 15: Curves of adaptive gains $\hat{\lambda}_{i}(i=2,3$, and 4$)$ in scenario 2.

from the rapidly decreasing of $r$. Thus, the disturbance observers are disabled if $r<r^{*}$, where $r^{*}$ is a predesigned parameter by trial and error.

Remark 11. Based on the above simulation results and analysis, it is apparent that the proposed AISMB-FTDO guidance law can provide better performance. Firstly, the comparisons between these three methods can verify the superiority of proposed FTDO. As shown in Figures 7 and 14 , it is obvious that the convergence time of SMO and 


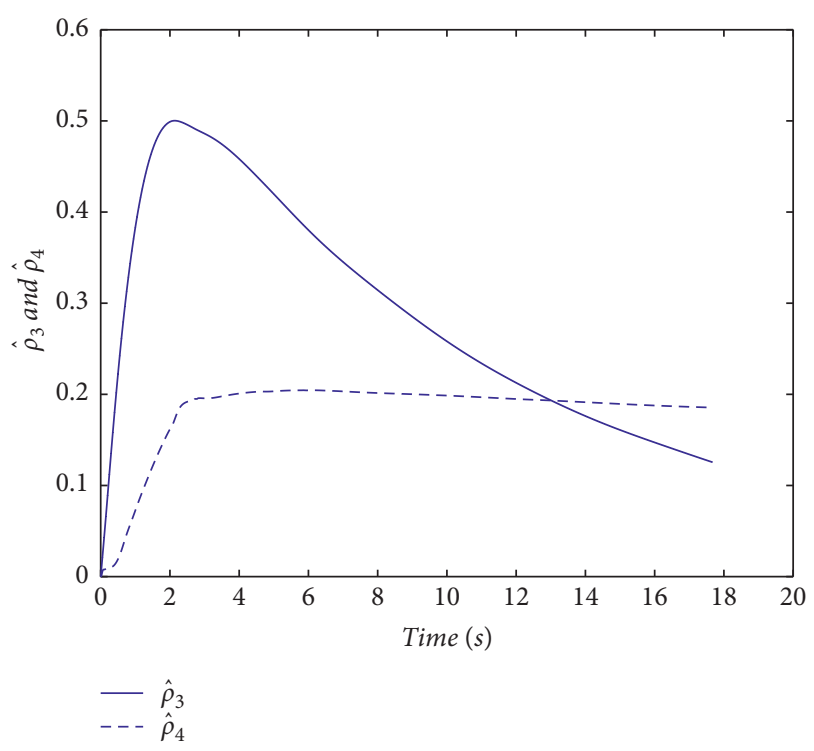

Figure 16: Curves of adaptive parameters $\widehat{\rho}_{j}(j=3$ and 4$)$ in scenario 2 .

FTDO is smaller than that of ESO. Besides, we can see from Figures 7 and 14 that the curves of FTDO are smoother than SMO since SMO causes serious chattering problem. Secondly, as shown in Tables 3 and 4, the control energy of SMB-ESO is much larger than that of AISMB-FTDO. For this phenomenon, one of the main reasons is that the proposed adaptive gain regulating laws exhibit well during the interception process. Finally, compared with traditional backstepping method in SMB-ESO, the adaptive rules are employed to handle the "differential explosion" problem of the virtual control input in the design process. Therefore, above analysis will further imply the contributions of our study.

\section{Conclusions}

This paper investigates the interception problem for a missile intercepting a target with impact angle constraint and autopilot dynamics. To solve this problem, we propose a FTDO-based adaptive finite time guidance law. The employed FTDO can achieve fast estimation for the lumped disturbance, and the proposed adaptive guidance laws $\widehat{\lambda}_{i}(i=$ 2,3 , and 4) can adjust the guidance gains accordingly. Besides, the "explosion of complexity" problem inherent in the conventional backstepping is avoided by utilizing the adaptive laws $\hat{\rho}_{i}(i=3$ and 4$)$. Furthermore, the simulation results clearly demonstrate the effectiveness of the proposed composite guidance law for intercepting the highly maneuvering target. In addition, we will further consider the guidance law design with control faults, sensor noises, and LOS limitations in our future study.

\section{Data Availability}

The data used to support the findings of this study are available from the corresponding author upon request.

\section{Conflicts of Interest}

The authors declare that they have no conflicts of interest.

\section{Acknowledgments}

This work was supported by National Natural Science Foundation of China (Grant nos. 91216304 and 61803357).

\section{References}

[1] J. Guo, Y. Li, and J. Zhou, "A new continuous adaptive finite time guidance law against highly maneuvering targets," Aerospace Science and Technology, vol. 85, pp. 40-47, 2019.

[2] Z. T. Cheng, W. Hao, and W. Bo, "Fixed-time convergent guidance law with impact angle control," Complexity, vol. 2020, p. 9, 2020.

[3] E. J. Ohlmeyer and C. A. Phillips, "Generalized vector explicit guidance," Journal of Guidance, Control, and Dynamics, vol. 29, no. 2, pp. 261-268, 2006.

[4] Y. Ulybyshev, "Terminal guidance law based on proportional navigation," Journal of Guidance, Control, and Dynamics, vol. 28, no. 4, pp. 821-824, 2005.

[5] D. Ghose, "Capture region for true proportional navigation guidance with nonzero miss-distance," Journal of Guidance, Control, and Dynamics, vol. 17, no. 3, pp. 627-628, 1994.

[6] T.-H. Kim, B.-G. Park, and M.-J. Tahk, "Bias-shaping method for biased proportional navigation with terminal-angle constraint," Journal of Guidance, Control, and Dynamics, vol. 36, no. 6, pp. 1810-1816, 2013.

[7] Y. Pin-Jar Yuan and H. Shih-Che Hsu, "Solutions of generalized proportional navigation with maneuvering and nonmaneuvering targets," IEEE Transactions on Aerospace and Electronic Systems, vol. 31, no. 1, pp. 469-474, 1995.

[8] C.-K. Ryoo, H. Cho, and M.-J. Tahk, "Optimal guidance laws with terminal impact angle constraint," Journal of Guidance, Control, and Dynamics, vol. 28, no. 4, pp. 724-732, 2005.

[9] D. Zhou, C. Mu, and W. Xu, "Adaptive sliding-mode guidance of a homing missile," Journal of Guidance, Control, and Dynamics, vol. 22, no. 4, pp. 589-594, 1999.

[10] S. Xiong, W. Wang, S. Song, S. Wang, and C. Lai, "Extended state observer based impact angle constrained guidance law for maneuvering target interception," Proceedings of the Institution of Mechanical Engineers-Part G: Journal of Aerospace Engineering, vol. 229, no. 14, pp. 2589-2607, 2015.

[11] B. Jiang, H. R. Karimi, S. Yang, C. Gao, and Y. Kao, "Observerbased adaptive sliding mode control for nonlinear stochastic markov jump systems via T-S fuzzy modeling: applications to robot arm model," IEEE Transactions on Industrial Electronics, vol. 68, no. 1, pp. 466-477, 2021.

[12] Z. Hou, L. Liu, and Y. Wang, "Time-to-go estimation for terminal sliding mode based impact angle constrained guidance," Aerospace Science and Technology, vol. 71, pp. 685-694, 2017.

[13] R. Chang-Kyung Ryoo, C. Hangju Cho, and T. Min-Jea Tahk, "Time-to-go weighted optimal guidance with impact angle constraints," IEEE Transactions on Control Systems Technology, vol. 14, no. 3, pp. 483-492, 2006.

[14] B. Jiang and C.-C. Gao, "Decentralized adaptive sliding mode control of large-scale semi-Markovian jump interconnected systems with dead-zone input," IEEE Transactions on Automatic Control, p. 1, 2021.

[15] Y. B. Shtessel, I. A. Shkolnikov, and A. Levant, "Guidance and control of missile interceptor using second-order sliding 
modes," IEEE Transactions on Aerospace and Electronic Systems, vol. 45, no. 1, pp. 110-124, 2009.

[16] B. Ebrahimi, M. Bahrami, and J. Roshanian, "Optimal slidingmode guidance with terminal velocity constraint for fixedinterval propulsive maneuvers," Acta Astronautica, vol. 62, no. 10-11, pp. 556-562, 2008.

[17] D. Zhou, S. Sun, and K. L. Teo, "Guidance laws with finite time convergence," Journal of Guidance, Control, and Dynamics, vol. 32, no. 6, pp. 1838-1846, 2009.

[18] S. P. Bhat and D. S. Bernstein, "Finite-time stability of continuous autonomous systems," SIAM Journal on Control and Optimization, vol. 38, no. 3, pp. 751-766, 2000.

[19] Y. Zhang, M. Sun, and Z. Chen, "Finite-time convergent guidance law with impact angle constraint based on slidingmode control," Nonlinear Dynamics, vol. 70, no. 1, pp. 619-625, 2012.

[20] S. R. Kumar, S. Rao, and D. Ghose, "Nonsingular terminal sliding mode guidance with impact angle constraints," Journal of Guidance, Control, and Dynamics, vol. 37, no. 4, pp. 1114-1130, 2014.

[21] L. Y. Zang, D. F. Lin, and Y. Ji, "Nonsingular continuous finite-time convergent guidance law with impact angle constraints," International Journal of Aerospace Engineering, vol. 2019, Article ID 6024240, 13 pages, 2019.

[22] J. Song, S. Song, and H. Zhou, "Adaptive nonsingular fast terminal sliding mode guidance law with impact angle constraints," International Journal of Control, Automation and Systems, vol. 14, no. 1, pp. 99-114, 2016.

[23] S. Xu, M. Gao, D. Fang, Y. Wang, and B. Li, “A novel adaptive second-order nonsingular terminal sliding mode guidance law design," Proceedings of the Institution of Mechanical Engineers-Part G: Journal of Aerospace Engineering, vol. 234, no. 16, pp. 2263-2273, 2020.

[24] D. Zhou and B. Xu, "Adaptive dynamic surface guidance law with input saturation constraint and autopilot dynamics," Journal of Guidance, Control, and Dynamics, vol. 39, no. 5, pp. 1155-1162, 2016.

[25] S. Sun, D. Zhou, and W.-t. Hou, "A guidance law with finite time convergence accounting for autopilot lag," Aerospace Science and Technology, vol. 25, no. 1, pp. 132-137, 2013.

[26] I. Rusnak and L. Meir, "Modern guidance law for high-order autopilot," Journal of Guidance, Control, and Dynamics, vol. 14, no. 5, pp. 1056-1058, 2015.

[27] S. He, D. Lin, and J. Wang, "Robust terminal angle constraint guidance law with autopilot lag for intercepting maneuvering targets," Nonlinear Dynamics, vol. 81, no. 1-2, pp. 881-892, 2015.

[28] Z. Zhao, C. Li, J. Yang, and S. Li, "Output feedback continuous terminal sliding mode guidance law for missile-target interception with autopilot dynamics," Aerospace Science and Technology, vol. 86, pp. 256-267, 2019.

[29] Y.-J. Wu, J.-X. Zuo, and L.-H. Sun, “Adaptive terminal sliding mode control for hypersonic flight vehicles with strictly lower convex function based nonlinear disturbance observer," ISA Transactions, vol. 71, pp. 215-226, 2017.

[30] S. Gao, "Extended-state-observer-based collision-free guidance law for target tracking of autonomous surface vehicles with unknown target dynamics," Complexity, vol. 2018, Article ID 4154670, 10 pages, 2018.

[31] S. He, J. Wang, and D. Lin, "Composite guidance laws using higher order sliding mode differentiator and disturbance observer," Proceedings of the Institution of Mechanical Engineers-Part G: Journal of Aerospace Engineering, vol. 229, no. 13, pp. 2397-2415, 2015.
[32] F. Yang and G. Xia, "A finite-time 3D guidance law based on fixed-time convergence disturbance observer," Chinese Journal of Aeronautics, vol. 33, no. 4, pp. 1299-1310, 2020.

[33] M. Basin, C. B. Panathula, and Y. Shtessel, "Multivariable continuous fixed-time second-order sliding mode control: design and convergence time estimation," IET Control Theory \& Applications, vol. 11, pp. 1104-1111, 2017.

[34] Z. Zuo, "Non-singular fixed-time terminal sliding mode control of non-linear systems," IET Control Theory \& Applications, vol. 9, no. 4, pp. 545-552, 2014.

[35] M. Basin, P. Yu, and Y. Shtessel, "Finite-and fixed-time differentiators utilising HOSM techniques," IET Control Theory \& Applications, vol. 11, no. 8, pp. 1144-1152, 2017.

[36] Z. Zuo, "Fixed-time consensus tracking for multi-agent systems with high-order integrator dynamics," IEEE Transactions on Automatic Control, vol. 63, 2017.

[37] T. Wu, "Quantized fixed-time fault-tolerant attitude control for hypersonic reentry vehicles," Applied Mathematical Modelling, vol. 98, 2021.

[38] M. V. Basin, P. Yu, and Y. B. Shtessel, "Hypersonic missile adaptive sliding mode control using finite- and fixed-time observers," IEEE Transactions on Industrial Electronics, vol. 65, no. 1, pp. 930-941, 2018.

[39] S. R. Kumar, S. Rao, and D. Ghose, "Sliding-mode guidance and control for all-aspect interceptors with terminal angle constraints," Journal of Guidance, Control, and Dynamics, vol. 35, no. 4, pp. 1230-1246, 2012.

[40] S. P. Bhat and D. S. Bernstein, "Continuous finite-time stabilization of the translational and rotational double integrators," IEEE Transactions on Automatic Control, vol. 43, no. 5, pp. 678-682, 1998.

[41] A. Polyakov, "Nonlinear feedback design for fixed-time stabilization of Linear Control Systems," IEEE Transactions on Automatic Control, vol. 57, no. 8, pp. 2106-2110, 2012.

[42] Y. Hong, Y. Xu, and J. Huang, "Finite-time control for robot manipulators," Systems \& Control Letters, vol. 46, no. 4, pp. 243-253, 2002.

[43] H. Pan, W. Sun, H. Gao, and X. Jing, "Disturbance observerbased adaptive tracking control with actuator saturation and its application," IEEE Transactions on Automation Science and Engineering, vol. 13, no. 2, pp. 868-875, 2016.

[44] X. Wang, J. Guo, S. Tang, and S. Qi, "Fixed-time disturbance observer based fixed-time back-stepping control for an airbreathing hypersonic vehicle," ISA Transactions, vol. 88, pp. 233-245, 2019.

[45] Z.-G. Zhou, "Prescribed performance fixed-time tracking control for a class of second-order nonlinear systems with disturbances and actuator saturation," International Journal of Control, vol. 94, pp. 1-12, 2019. 